\title{
IN-SYNC. VIII. Primordial Disk Frequencies in NGC 1333, IC 348, and the Orion A Molecular Cloud
}

\author{
Yuhan Yao ${ }^{1,2}$ (1), Michael R. Meyer ${ }^{3}$ (1), Kevin R. Covey ${ }^{4}$, ${ }^{\text {, Jonathan C. } \operatorname{Tan}^{5,6} \text {, and Nicola Da Rio }}{ }^{6}$ \\ ${ }^{1}$ Cahill Center for Astrophysics, California Institute of Technology, Pasadena, CA 91125, USA \\ ${ }^{2}$ Department of Astronomy, Peking University, Yi He Yuan Lu 5, Hai Dian District, Beijing 100871, People's Republic of China \\ ${ }^{3}$ Department of Astronomy, University of Michigan, Ann Arbor, MI 48109, USA \\ ${ }^{4}$ Department of Physics \& Astronomy, Western Washington University, Bellingham, WA 98225, USA \\ ${ }^{5}$ Department of Space, Earth and Environment, Chalmers University of Technology, Gothenburg, Sweden \\ ${ }^{6}$ Department of Astronomy, University of Virginia, Charlottesville, VA 22904, USA \\ Received 2018 July 26; revised 2018 October 14; accepted 2018 October 15; published 2018 December 13
}

\begin{abstract}
In this paper, we address two issues related to primordial disk evolution in three clusters (NGC 1333, IC 348, and Orion A) observed by the INfrared Spectra of Young Nebulous Clusters (IN-SYNC) project. First, in each cluster, averaged over the spread of age, we investigate how disk lifetime is dependent on stellar mass. The general relation in IC 348 and Orion A is that primordial disks around intermediate-mass stars $\left(2-5 M_{\odot}\right)$ evolve faster than those around loss-mass stars $\left(0.1-1 M_{\odot}\right)$, which is consistent with previous results. However, considering only low-mass stars, we do not find a significant dependence of disk frequency on stellar mass. These results can help to better constrain theories on gas giant planet formation timescales. Second, in the Orion A molecular cloud, in the mass range of $0.35-0.7 M_{\odot}$, we provide the most robust evidence to date for disk evolution within a single cluster exhibiting modest age spread. By using surface gravity as an age indicator and employing $4.5 \mu \mathrm{m}$ excess as a primordial disk diagnostic, we observe a trend of decreasing disk frequency for older stars. The detection of intracluster disk evolution in NGC 1333 and IC 348 is tentative, since the slight decrease of disk frequency for older stars is a less than $1 \sigma$ effect.
\end{abstract}

Key words: protoplanetary disks - stars: formation - stars: pre-main sequence

Supporting material: machine-readable table

\section{Introduction}

Circumstellar disks are a natural consequence of the conservation of angular momentum during the collapse of star-forming molecular clouds (Williams \& Cieza 2011). Excesses above the stellar photosphere at short infrared (IR) wavelengths $(2-8 \mu \mathrm{m})$ trace dust in the inner disk (Hartigan et al. 1995). The excesses generally decrease and finally disappear as the disks evolve. The disappearance of gas requires accretion into the star, accretion into giant planets, or photoevaporation by the radiation from the central star (Hartmann 2009). The timescale of gas dispersal has been found to be similar to that of dust from measurements of accretion indicators (Fedele et al. 2010). This timescale around typical young stars is found to be $\sim 3 \mathrm{Myr}$ (Haisch et al. 2001; Hernández et al. 2007; Mamajek 2009).

There is clear evidence that disk evolution is dependent on stellar mass. Previous studies show that gas-rich disk frequencies around solar-to-higher-mass stars are lower than those around low-mass stars (Hillenbrand et al. 1998; Hernández et al. 2005; Carpenter et al. 2006; Ribas et al. 2014, 2015; Yasui et al. 2014), which indicates that disks around early-type stars evolve more quickly due to more efficient disk dispersal (Kennedy \& Kenyon 2009). Lada et al. (2006, hereafter Lada06) suggested a maximum disk frequency around stars with spectral type K6-M2 in the partially embedded cluster IC 348 . Dahm \& Hillenbrand (2007) and Hernández et al. (2007) found similar peaks in NGC 2362 and the Orion OB1 association, respectively. However, the observed decline of disk frequency for the very lowest-mass stars was not conclusive due to (1) poisson error; (2) observational bias: at a fixed wavelength (temperature), the smaller solid angle of disks around low-mass stars makes it more difficult to distinguish disk emission relative to stellar emission; (3) inner disk hole size and system inclination: high inclinations or large holes will make it harder to detect disk excesses, especially for low-mass stars (Hillenbrand et al. 1998).

The lifetime of gas-rich disks sets a limit to the timescale available for gas giant planet formation (from substantial amounts of gas accreted onto the planetary core; Meyer et al. 2007; Currie et al. 2009). Given the dependence of disk fraction on stellar mass, less time is available for gas giant planet formation around more massive stars. However, a competing effect is that disk mass also increases with stellar mass (Andrews et al. 2013), so processes dependent on disk mass surface density and orbital timescale may proceed faster around stars of higher mass (Meyer 2009). Observations have shown hints of a higher giant planet occurrence rate around more massive stars (Johnson et al. 2010; Bowler 2016). Hence, examining trends in disk lifetime versus stellar age as a function of stellar mass will put a major constraint on theories of planet formation.

If the age spread of young stellar objects (YSOs) in a young cluster is small enough that these YSOs can be assumed to be coeval, and if their spectral types are known, one can separate the effects of the intrinsic color of the photosphere, reddening, and intrinsic color excess due to the presence of the disk (Meyer et al. 1997). Then disk frequency can be calculated in an unbiased way for an extinction-limited sample as the fraction of stars with excess at a certain wavelength as a function of spectral type (stellar mass).

Thanks to the INfrared Spectra of Young Nebulous Clusters (IN-SYNC) program, more than 3000 stars in NGC 1333, IC 348, 
and the Orion A molecular cloud have been observed with the Apache Point Observatory Galactic Evolution Experiment (APOGEE) project from the third Sloan Digital Sky Survey (Eisenstein et al. 2011, SDSS-III). These stars have effective temperatures $\left(T_{\text {eff }}\right)$ and surface gravities $(\log g)$ determined with a consistent modeling approach using APOGEE's high-resolution $H$-band spectra (Cottaar et al. 2014), and therefore provide good samples without any systematic discrepancies in terms of stellar parameters. In this paper, using $\sim 2000$ of these stars, we address two issues in each of the three clusters. On one hand, by taking a snapshot of each cluster averaged over the spread of age, we investigate how disk frequency is dependent on stellar mass, with particular focus on the low-mass range $\left(0.1-1.5 M_{\odot}\right)$. On the other hand, since Cottaar et al. (2014) and Da Rio et al. (2016) have detected the intrinsic age spreads on the order of $\sim \mathrm{Myr}$ in these clusters, we are motivated to study disk evolution within every single cluster by using $\log g$ as an age indicator. This is a novel approach to disk evolution problems without having to assume that each cluster is somehow representative of a global population.

This paper is organized as follows. The next section provides an assessment of the representativeness of our sample. We outline our disk diagnostic in Section 3. Evidence of disk evolution related to stellar mass and stellar age are illustrated in Sections 4 and 5, respectively. A conclusion is given in Section 6. The sample used in this paper can be found in the Appendix.

\section{Sample Properties}

In this section, we assess the degree to which our sample of stars is considered representative of the entire population of cluster members using color-magnitude diagram (CMD) methods. First, in Section 2.1, we briefly describe the INSYNC survey, and the properties of each cluster being observed. Then, in Section 2.2, we make some initial cuts to the observed IN-SYNC sample to choose a subset of stars for data analysis. After that, we individually determine the representative mass range under an extinction limit in each cluster in Section 2.3. Last, in Section 2.4, we point out some caveats in our assessment.

\subsection{The IN-SYNC Survey}

The star-forming regions targeted by the IN-SYNC program are IC 348 and NGC 1333 in the Perseus molecular cloud, the Orion A molecular cloud, and NGC 2264. Since a relatively small number of sources were observed in NGC 2264, we do not include this cluster in our study. The APOGEE spectrograph covers the spectral range from 1.51 to $1.70 \mu \mathrm{m}$ with a spectral resolution of $\sim 22,500$. It can also observe up to 300 targets in a three-degree-diameter field of view (FoV) at the same time (Majewski et al. 2017). However, it cannot simultaneously observe stars within $71 . " 5$ of each other due to fiber collision. Therefore, multiple plates were drilled to cover the densest regions.

Target selection for spectroscopic survey of the Perseus fields was described in detail in Foster et al. (2015) and Cottaar et al. (2015). In summary, potential targets were compiled from candidate or confirmed members selected via signatures of youth. Observations were designed to maximize the completeness for sources with $8<H<12.5$. For these bright sources, high priority was further sorted according to their extinctioncorrected $H$-band magnitudes.

The Orion A molecular cloud is the nearest known massive stellar nursery. The large structure includes the Upper Sword, NGC 1977, Orion Molecular Cloud 2/3 region (OMC-2/3), Orion Nebula Cluster (ONC), ८ Ori (also called NGC 1980), and the low-density L1641 region (Muench et al. 2008). Targets for the IN-SYNC Orion survey have been primarily selected from known or candidate members in ONC or L1641, accompanied with bright 2MASS sources with unknown membership. Unlike the Perseus survey, observations were only conducted for sources with $H<12.5$. Details of the observing strategy are described by Da Rio et al. (2016).

To assess the representative mass range, we need to know the age and distance of each cluster. The adopted values are presented in Table 1. For each cluster, we use the most commonly cited age estimates for consistency with other works concerning disk evolution. Distance estimates are derived by cross-matching cluster members (compiled in Section 2.2.1) with the Gaia second data release (Gaia Collaboration et al. 2018, hereafter Gaia DR2). In each cluster, we calculate the $25 \mathrm{th}, 50 \mathrm{th}$, and 75 th percentiles of the distance distribution, as are shown in the third column of Table 1. The median distance in each cluster will be adopted for assessing the representative mass range. However, we also provide results corresponding to a tolerant distance estimation (the 25 th percentile) and a restrictive distance estimation (the 75 th percentile). See Section 2.3 for details.

\subsection{Sample Selection}

Here we present the procedures to select a sample of stars for data analysis from the observed targets.

\subsubsection{Membership}

To identify cluster members from the observed sources, we first use previous membership studies to select YSO candidates. Then we make restrictions on their Gaia DR2 distances and proper motions to reduce possible contamination from field stars or background giants.

Previous Studies. Since target selection for the IN-SYNC survey was performed about five years ago, some recently identified cluster members were not included in the initial catalog. Since unfilled APOGEE fibers were assigned to other sources within the same field, however, some were serendipitously observed. On the other hand, some stars in the input catalog may display spectral signatures of youth, but are recently rejected as non-members by proper motion measurements. Therefore, in Perseus, to select cluster members from the observed stars, we first combine our input catalog with the updated census of NGC 1333 and IC 348 presented by Luhman et al. (2016, Table 1 and Table 2), and exclude those with

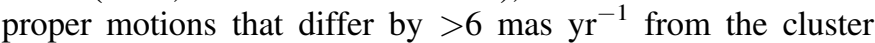
median (Table 3 of Luhman et al. 2016). After that, we crossmatch the compiled catalog with the IN-SYNC targets to identify bona fide members observed by IN-SYNC. This identifies 104 members in NGC 1333 and 372 in IC 348.

Membership identification for Orion is different. In total, 2691 stars were observed in the Orion A region; 1709are previously known members confirmed by spectroscopy, X-ray emission, or infrared excess; among the remaining 982 sources, a previous work in the IN-SYNC program (Da Rio et al. 2016) 
Table 1

Adopted Values for Each Cluster

\begin{tabular}{|c|c|c|c|c|c|c|}
\hline \multirow[t]{2}{*}{ Cluster } & \multirow{2}{*}{$\begin{array}{l}\text { Mean Age } \\
\text { (Myr) }\end{array}$} & \multirow{2}{*}{$\begin{array}{l}\text { Distance } \\
\quad(\mathrm{pc})\end{array}$} & \multirow{2}{*}{$\begin{array}{c}\text { Representative } \\
\text { Mass Range }\left(M_{\odot}\right)\end{array}$} & \multirow{2}{*}{$\begin{array}{l}\text { Extinction Limit } \\
\text { (mag) }\end{array}$} & \multicolumn{2}{|c|}{ SpType } \\
\hline & & & & & $\overline{2.2 M_{\odot}}$ & $\overline{5 M_{\odot}}$ \\
\hline \multirow[t]{2}{*}{ NGC 1333} & $1-2$ (a) & 282.3 & $0.1-1.5$ & $A_{J}<10$ & K3 & B5 \\
\hline & & 329.3 & $0.1-1.5$ & & & \\
\hline \multirow[t]{2}{*}{ IC 348} & 3 (b) & 304.2 & $0.23-1.5$ & $A_{J}<6$ & K1 & B5 \\
\hline & & 324.2 & $0.25-1.5$ & & & \\
\hline \multirow[t]{3}{*}{ Orion A } & $2(\mathrm{c})$ & 380.0 & $0.31-1.5$ & & K2 & B5 \\
\hline & & 396.7 & $0.34-1.5$ & $A_{J}<3$ & & \\
\hline & & 415.1 & $0.36-1.5$ & & & \\
\hline
\end{tabular}

Note. Age references: (a) Lada et al. (1996), (b) Muench et al. (2007), (c) Muench et al. (2008). Distances are the 25th, 50th, and 75th percentiles of the Gaia DR2 distance distribution of cluster members. The representative mass range is given at a certain extinction limit.

determines whether or not they are member candidates. The authors place all the sources in a number of planes (including the CMD $H-(J-H)$ plane, the H-R diagram, the $\log g-T_{\text {eff }}$ plane, the position-velocity plane), and compare the location occupied by each of them with the region occupied by the 1709 known members, since the latter generally occupy wellconfined regions. For each star with no membership, in each plane, Da Rio et al. (2016) consider the 50 closest sources to the star, and count among the 50 sources the fraction that are previously known members. This value is a basic indicator of its membership probability. The method identified 383 new candidate members, with $599(=982-383)$ stars considered to be non-members. We remove these 599 stars and leave 2092 in the Orion A sample.

Gaia Distances and Proper Motions. Section 5 of Da Rio et al. (2016) provides a discussion of the limitation of their method. In brief, newly identified members are only candidates, but not confirmed members. Possible contamination can be further eliminated if distances and proper motions are known. Therefore, we cross-match the 2092 sources in Orion A with the Gaia DR2 catalog; 1900 of them have the "astrometric global iterative solution" (AGIS) including position, parallax, and proper motion. Their proper motions are shown in Figure 1, color-coded according to distances. The colormap is adjusted such that the deeper the color, the further the star is from the median distance of $396.7 \mathrm{pc}$ (Table 1 ). We reject objects either with motions that differ by more than 6 mas $\mathrm{yr}^{-1}$ from the median of proper motion (beyond the dotted circle in Figure 1), or with distances that differ by more than $200 \mathrm{pc}$

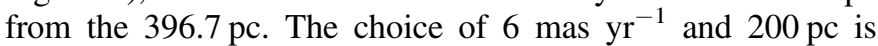
based on inspection of Figure 1. Of the 1900 candidates, 1645 survive these cuts.

We demonstrate the importance of this step in Figure 2. The distribution of all of the 2092 member candidates on the $\log g-T_{\text {eff }}$ plane is shown in the upper panel. 1900 targets with Gaia DR2 data are color-coded by distances, while the 192 sources without Gaia data are shown as yellow dots. In Section 2.2.2, we will only select targets with $3000 \mathrm{~K}<$ $T_{\text {eff }}<4750 \mathrm{~K}$. Hence, only sources in this temperature range are displayed. At the bottom right of this panel, there is clearly a cluster of deep purple dots with very high $\log g$ and small distances. They are probably contaminants of field stars. There are also a few distant stars locating around $T_{\text {eff }} \approx 4300 \mathrm{~K}$,

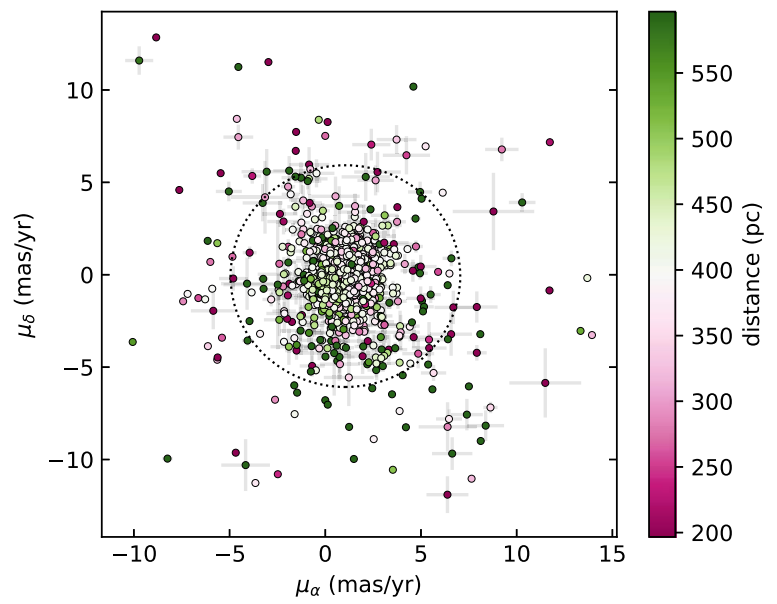

Figure 1. Proper motions of 1900 member candidates in Orion A, color-coded according to distances. The dotted line marks a circle with a radius of 6 mas $\mathrm{yr}^{-1}$ from the proper motion median $\left(\mu_{\alpha}=1.05, \mu_{\delta}=-0.08\right)$.

$\log g \approx 2.9$, which should be contaminants of background red giant stars.

The lower panel of Figure 2 shows distribution of the 1645 targets surviving cuts on proper motion and distance, as well as the 192 sources without Gaia data. Most contaminations from both field stars and background giants are excluded. Although we are unable to make cuts on the 192 yellow dots, they generally lie along the majority of cluster members on the $\log g-T_{\text {eff }}$ diagram, and may contain very few contaminants. Therefore, we choose not to exclude any sources from them, and the number of sources retained in Orion $\mathrm{A}$ is reduced to $1837(=1645+192)$.

To make sure that our analysis is consistent in all of the three clusters, we also apply the procedures stated above in the other two clusters. After that, the number of sources decreases from 104 to 95 in NGC 1333, and from 372 to 324 in IC 348.

\subsubsection{Stellar Parameters}

All IN-SYNC spectra were modeled by a spectral fitting approach presented in Cottaar et al. (2014), which is suited for determining stellar parameters of young stars. In brief, observed spectra are modeled with a grid of "BT-Settl" synthetic spectra (Allard et al. 2012). Five free parameters are included in the fitting: $T_{\text {eff }}, \log g$, radial velocity $\left(v_{r}\right)$, rotational 


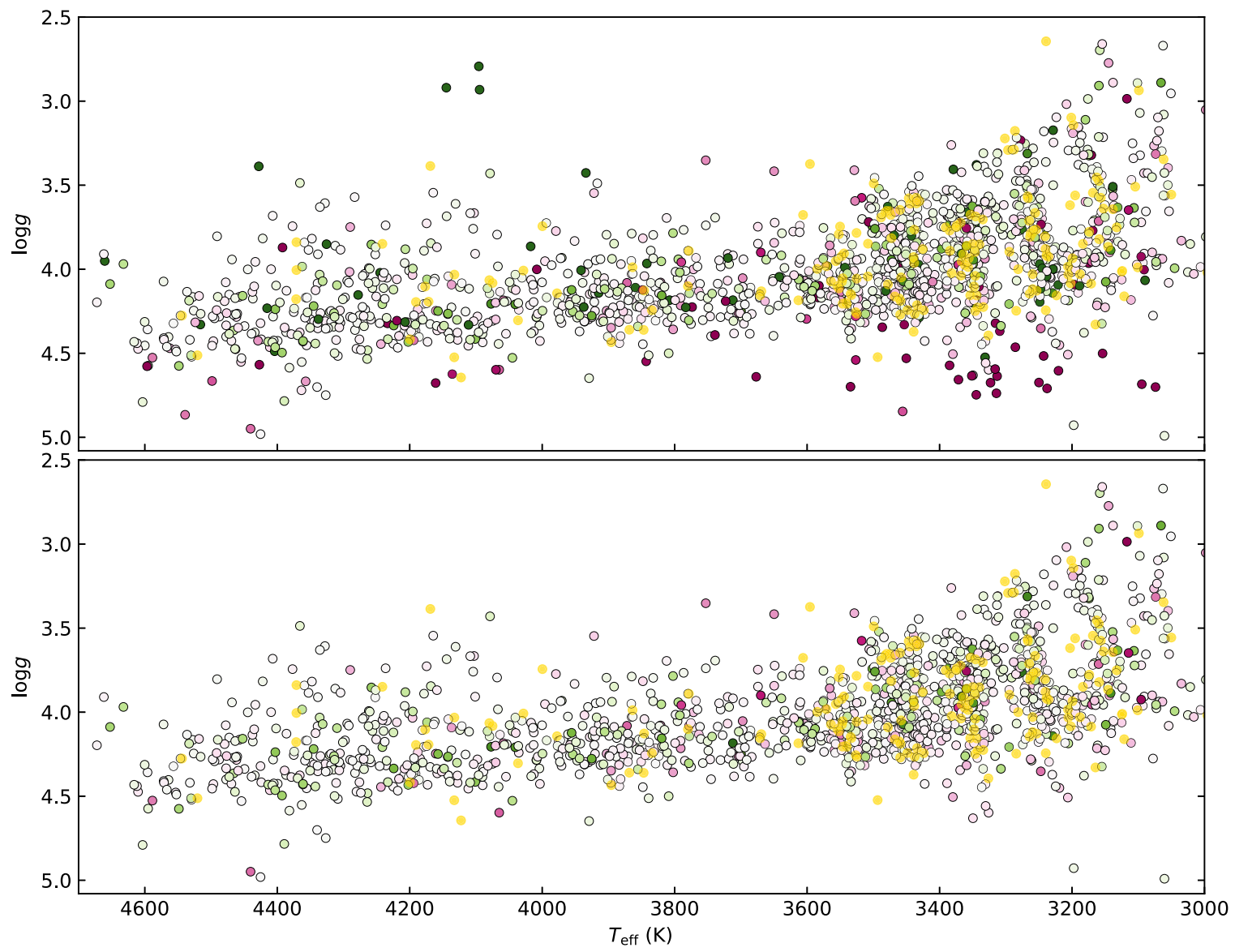

Figure 2. Upper panel: distribution of 2092 member candidates in Orion A on the $\log g-T_{\text {eff }}$ plane. 1900 targets with Gaia DR2 data are color-coded by distances (the color scale is the same as in Figure 1). 192 targets without Gaia data are shown as the yellow dots. Lower panel: distribution of 1837 accepted cluster members, including 1645 targets with Gaia data and 192 targets not in the Gaia catalog.

velocity $(v \sin i)$, and $H$-band veiling $\left(r_{H}\right)$. For targets with multiple observations, we adopt the average values determined as the weighted mean of the parameters measured from all epochs. Parameter uncertainties are initially estimated from a Markov Chain Monte Carlo (MCMC) simulation, and then inflated to match the epoch-to-epoch variability seen for the same star at different epochs.

Although spectra in all clusters were initially modeled by Cottaar et al. (2014), Da Rio et al. (2016) implemented changes to correct and optimize aspects of the code, and utilized the updated algorithm to model spectra of sources in Orion A. Results from the latter paper should supersede those from Cottaar et al. (2014). Unfortunately, we do not have the new parameters for sources in Perseus, so we still need to use Cottaar's parameters for NGC 1333 and IC 348. In Figure 3, we compare the $T_{\text {eff }}$ and $\log g$ determined by the two authors for sources in Orion A. In the upper panel, it is obvious that there is a systematic discrepancy of $T_{\text {eff }}$ determined by the two methods. This offset is most prominent near $T_{\text {eff }} \approx 3900 \mathrm{~K}$, where the Da Rio values are several hundred kelvins cooler than the Cottaar results. As Da Rio et al. (2016) found no such offset in their comparison to $T_{\text {eff }}$ estimates in the literature (see their Figure 3), we adopt the scale implied by the Da Rio results. To this end, we fit a spline function of $T_{\text {eff }}$ (Cottaar) versus $T_{\text {eff }}$ (Da Rio) to the data points, which is shown as the red line. Cottaar's temperatures of the Perseus sources are then scaled with this relation to bring them into agreement with Da Rio's. By inspection of the lower panel, we see no discrepancies for the surface gravity measurements. The data generally follow a linear relation of $\log g($ Cottaar $)=\log g$ (Da Rio). Therefore, for sources in Perseus, we do not make any scaling to the $\log g$ produced by Cottaar.

Cottaar et al. (2014) and Da Rio et al. (2016) also find that APOGEE- $T_{\text {eff }}>4750 \mathrm{~K}$ is less reliable due to the smaller number of features in $H$-band spectra for hotter stars. Therefore, we only retain stars with $3000 \mathrm{~K}<T_{\text {eff }}<4750 \mathrm{~K}$. This subset of cluster members should have accurately determined $T_{\text {eff }}$ for data analysis. After this temperature cut, the number of stars decreases from 95 to 75 in NGC 1333, from 324 to 263 in IC 348, and from 1837 to 1702 in Orion A. The average age of each cluster ranges from 1 to $3 \mathrm{Myr}$ (Table 1). According to the Baraffe et al. (1998, hereafter BCAH98) premain-sequence (PMS) evolutionary models, for stars with ages between 1 and $3 \mathrm{Myr}, T_{\text {eff }}=3000 \mathrm{~K}(4750 \mathrm{~K})$ corresponds to $\sim 0.1 M_{\odot}\left(1.5 M_{\odot}\right)$ stars.

\subsubsection{Photometry}

IRAC. We utilize infrared photometry from online archives of the Spitzer Infrared Array Camera (IRAC) instrument, which includes four bands at 3.6, 4.5, 5.8, and $8.0 \mu \mathrm{m}$. To obtain an idea of IRAC's detectability, we calculate the expected photospheric IRAC flux of a $T_{\text {eff }}=3000 \mathrm{~K}, \log g=4.0$ star by convolving synthetic "BT-Settl" photospheres (Allard et al. 2012) with the response curve of IRAC filters. The choice of $T_{\text {eff }}$ and $\log g$ is typical of a low-mass $\left(\sim 0.1 M_{\odot}\right)$ young star. 

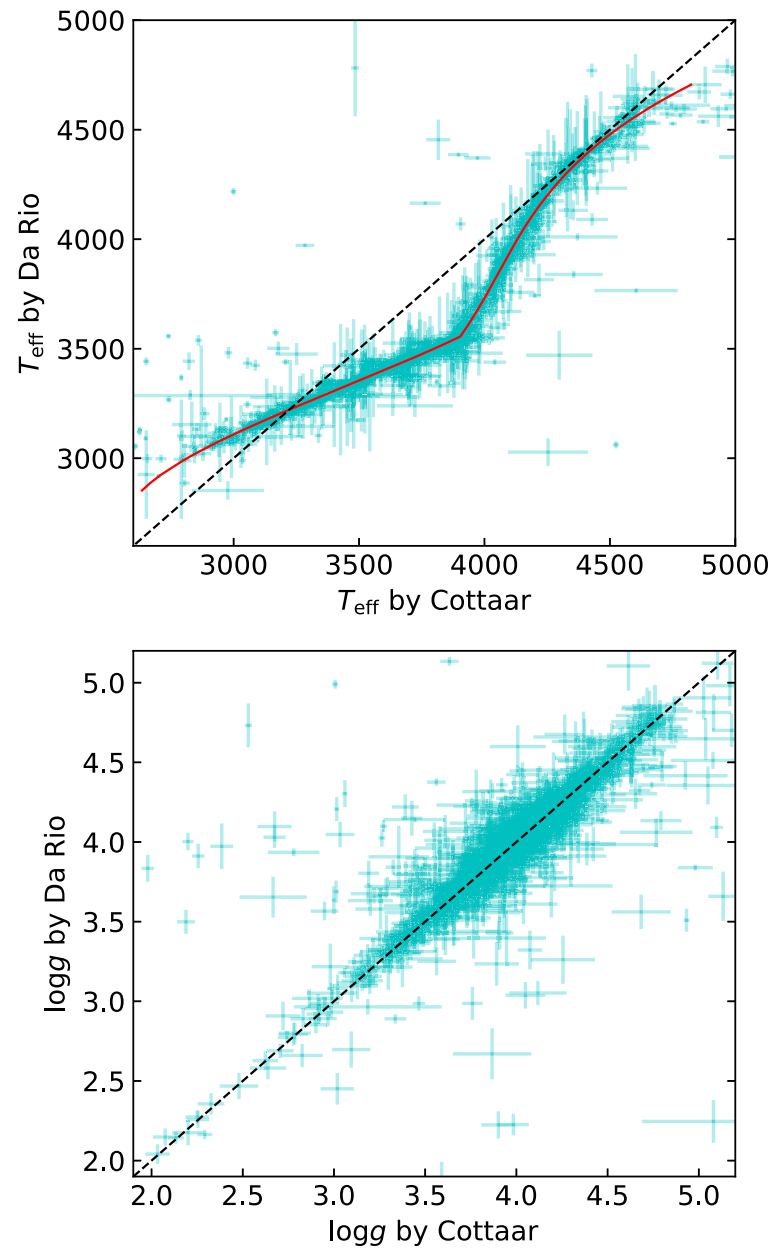

Figure 3. Comparison of $T_{\text {eff }}$ and $\log g$ determined for IN-SYNC Orion A targets by Cottaar et al. (2014) and Da Rio et al. (2016). The red line in the upper panel is a spline fit of the data points, while the dashed black lines in both panels are just diagonal lines of $y=x$.

Assuming a distance of $420 \mathrm{pc}$ and an extinction of $A_{J}=6$, this star would appear to be a source with $I_{1}=13.062$, $I_{2}=12.696, I_{3}=12.586, I_{4}=12.578$, well above the limit magnitude of IRAC. Since all sources in our sample are hotter $\left(3000 \mathrm{~K}<T_{\text {eff }}<4750 \mathrm{~K}\right)$, closer $(\sim 280-415 \mathrm{pc})$, and suffer from less extinction, they should be detected by IRAC even if there is no disk emission.

IRAC data for the Perseus clusters are obtained from the $\mathrm{c} 2 \mathrm{~d}$ Spitzer Legacy Project (Evans et al. 2003, 2009). ${ }^{7}$ Among the 75 stars in NGC 1333, 1 star (2MASS J03290915+3121445, $H=14.8$ ) has no data in the c2d catalog. The online image shows that it is only about $20^{\prime \prime}$ from the center of the nebula. It is suspected that the non-detection may arise from a technical issue. We remove this star, and retain 74 for further analysis. Among the 263 stars in IC 348, 17 are outside the FoV of the c2d survey. We retain the 246 sources within the FoV of the IRAC camera.

IRAC data for Orion A are obtained from the Spitzer Enhanced Imaging Products (SEIP) source list. ${ }^{8}$ To ensure high reliability, SEIP remove many observed sources by strict cuts in size, blending, signal-to-noise ratio, etc. Hence, in the most crowded region of ONC, the list is highly incomplete. We use

\footnotetext{
7 http://irsa.ipac.caltech.edu/data/SPITZER/C2D/

8 http://irsa.ipac.caltech.edu/data/SPITZER/Enhanced/SEIP/
}

the 3". 8 diameter aperture flux density (including band-filled fluxes when the IRAC source is extended). 1431 sources have extracted SEIP fluxes at $4.5 \mu \mathrm{m}$. We note that although IRAC data for another $\sim 100$ stars can be obtained from Megeath et al. (2012, the Spitzer Orion survey), we only retain the 1431 SEIP sources, because Megeath et al. (2012) only published sources with infrared excess, which means most (if not all) of these $\sim 100$ sources have disks. Thus, including them will bias our sample to stars with disks and render disk frequency higher than the true value.

2MASS. Three sources in IC 348 fail to be cross-matched with the Two Micron All Sky Survey (2MASS) all-sky point source catalog (Skrutskie et al. 2006). They are removed since 2MASS colors are needed for our estimates of extinction and color excess (see Section 3). The number of sources in the IC 348 sample decreases from 246 to 243.

\subsection{Representative Mass Range}

Bearing in mind that our sample is far from being complete at the low-mass end, in each cluster we study the range of stellar mass that is considered to be representative by our sample. Isochrones used in this section are calculated by BCAH98 with a convection mixing length of $\alpha=1.9$.

\subsubsection{NGC 1333}

The final sample of 74 sources in NGC 1333 are shown as the red dots in Figure 4. The 21 stars shown with blue crosses are also observed by IN-SYNC, but removed from data analysis. The red and blue add up to the 95 cluster members retained in Section 2.2.1. Pecaut \& Mamajek (2013) provide intrinsic color $(J-H)$ and $T_{\text {eff }}$ for $<30$ Myr old PMS stars. We convert $T_{\text {eff }}$ to stellar mass and $M_{J}$ using the BCAH98 isochrone of $1 \mathrm{Myr}$ stars. The black line in the upper panel of Figure 4 shows the expected $J$ versus $(J-H)$ isochrone adopting a median distance of $300.7 \mathrm{pc}$ for this cluster (see Table 1).

We use the following method to select possible cluster members not targeted by IN-SYNC. First of all, we collect all 2MASS sources within $10^{\prime}$ from the center of NGC 1333. Most cluster members and few field stars are expected to be inside of this circular field. The radius of $10^{\prime}$ is chosen based on inspection of the spatial distribution of cluster members. Among these 2MASS sources, targeted stars (red and blue) and known field stars tabulated in Luhman et al. (2016, Table 3) are removed, resulting in 221 sources. Then, to further exclude contamination of non-cluster members in the 221 sources, we cross-match them with Gaia DR2. For the 83 sources with proper motion and distance measurements, we reject stars

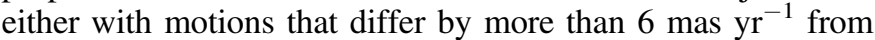
the median proper motion of cluster members $\left(\mu_{\alpha}=7.48\right.$, $\mu_{\delta}=9.89$, found in Section 2.2.1), or with distances smaller than $100.7(=300.7-200)$ pc or greater than $500.7(=300.7$ $+200) \mathrm{pc}$, where $300.7 \mathrm{pc}$ is the median distance of cluster members. The 23 sources that pass these cuts are shown as gray pluses in Figure 4. Note that this criterion is also adopted to reject contaminants in Section 2.2.1. The 138 2MASS sources without Gaia data are shown as green pluses.

To assess the representativeness of the used sample in NGC 1333 (red dots in Figure 4), we perform a two-sample 2D K-S test to test the null hypothesis that the used sample is drawn from the same distribution as that of the total cluster members. 

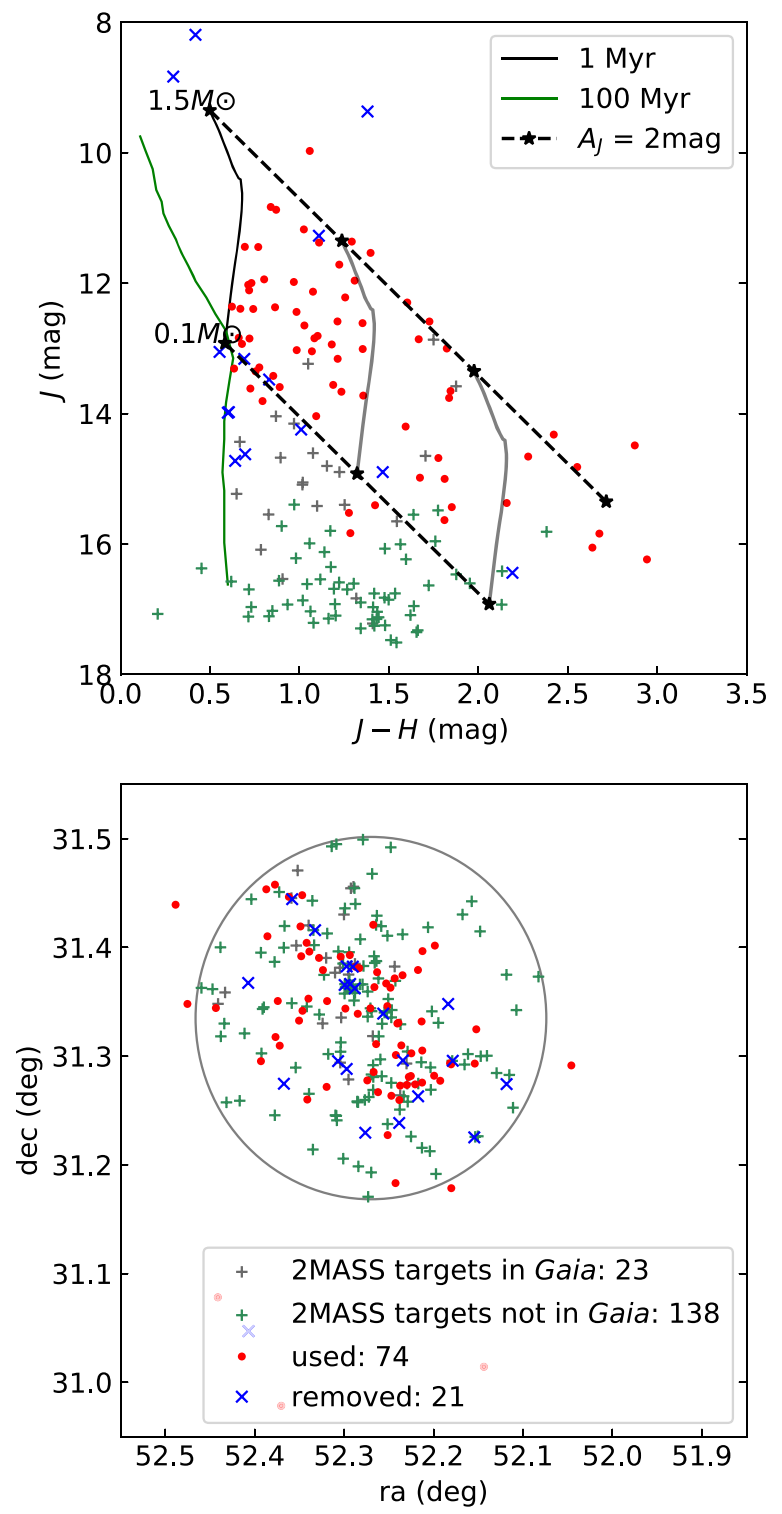

Figure 4. Upper panel: Color-magnitude diagram (CMD) of sources in NGC 1333. Overplotted isochrones are from BCAH98. 74 observed cluster members in our sample are shown as red dots; 21 observed members not in our sample are shown as blue crosses. Other 2MASS sources within $10^{\prime}$ from the star 2MASS J03290832+3120203are marked as pluses. Among them, the 138 sources without Gaia data are shown in green; the 23 sources with Gaia proper motion and distance measurements being consistent with being cluster members are shown in gray (see text). An extinction vector of $A_{J}=2 \mathrm{mag}$ is the length of the dashed black line between two black asterisks. Lower panel: spatial distribution of these sources.

Here we assume that the total cluster members can be represented by observed cluster members (red + blue) combined with other 2MASS stars within the adopted cluster radius (gray + green). The applicability of including these 2MASS sources in the K-S tests is discussed in Section 2.4.2.

This test is utilized to see if the used sample is uniformly selected from the total, or if it represents a subsample that is not consistent with the total. It is performed at a certain extinction limit of $A_{J}$, and in a certain mass range. In short, in the upper panel of Figure 4, in the corresponding zone of the CMD, the 2D K-S test ranges over every point on the $(J-H, J)$ plane to calculate the fraction of points (i.e., probability) from the two samples (red versus red+blue+gray+green) in each of the four

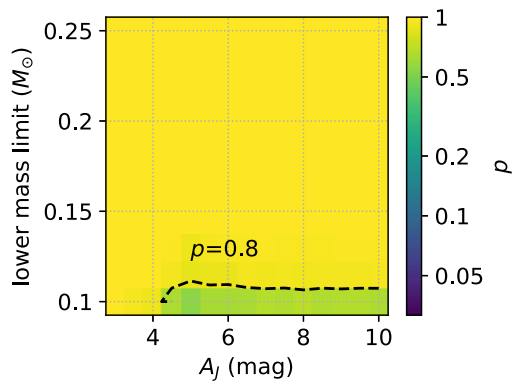

Figure 5. K-S test significance level $(p)$ for the null hypothesis that the extinction-limited, mass-limited NGC 1333 sample is uniformly drawn from the total of NGC 1333. The $X$-axis shows the extinction limit, while the $Y$-axis shows the lower mass limit. A contour of $p=0.8$ is shown as the dashed black line.

natural quadrants, in search of the maximum probability difference (defined as $D$ ) ranging both over data points and over quadrants (Press et al. 2002, Section 14.7).

To know if $D$ is statistically significant, we generate 1000 synthetic data sets by randomly choosing some points from the total (allowing repeat). Defining $N_{1}$ to be the number of points in the sample, and $N_{2}$ to be that in the total, each data set has $N_{1}$ points. We then compute $D$ for each synthetic data set, and count what fraction of the 1000 synthetic $D$ exceeds the $D$ from the real sample. This fraction is the significance level (p-value). ${ }^{9}$ One might consider a hypothesis suspect of $p<$ 0.05 , but here we adopt a conservative value of $p>0.2$ to insist that the null hypothesis is retained. The upper mass limit is set at $1.5 M_{\odot}$, and we let the lower mass limit vary from 0.1 to $0.25 M_{\odot}$. The extinction limit of $A_{J}$ changes from 3 to 10 , because among all of the 74 stars in the sample, the highest extinction is $A_{J}=9.2$ (see Section 3.2 for the determination of extinction). The resulting $p$ is shown as the color map in Figure 5. In the parameter space being investigated, the returning $p$-values are above 0.2 . We then conclude that our sample is representative for $0.1-1.5 M_{\odot}$ at $A_{J}=10$ in NGC 1333 .

\subsubsection{IC 348}

In Figure 6, we show the 243 stars in the final sample of IC 348 as the red dots. The 78 blue crosses are also observed cluster members that are removed from further analysis. The red and blue add up to 321 , which is not the 324 sources retained in Section 2.2.1, because 3 sources do not have 2MASS data. We convert $T_{\text {eff }}$ in Pecaut \& Mamajek (2013) to stellar mass and $M_{J}$ using the BCAH98 isochrone of $3 \mathrm{Myr}$ stars. The black line in the upper panel of Figure 6 shows the expected isochrone adopting a median distance of $324.2 \mathrm{pc}$ for this cluster (see Table 1).

Similar to what we have done for NGC 1333, 2MASS sources within $14^{\prime}$ from the center of IC 348 are collected. The radius of $14^{\prime}$ is also selected based on inspection of the lower panel of Figure 6 to include most cluster members and few field stars. Again, to reduce contamination, we remove known field stars tabulated in Luhman et al. (2016, Table 3), which gives us 708 sources to be cross-matched with Gaia. Among the 512

\footnotetext{
9 We have also computed $D$ using Equation (14.7.1) of Press et al. (2002), which evaluates if $\frac{\sqrt{N} D}{1+\sqrt{1+r^{2}}(0.25-0.75 / \sqrt{N})}$ is large enough to reject the null hypothesis, where $N=N_{1} N_{2} /\left(N_{1}+N_{2}\right)$ and $r$ is Pearson's coefficient of correlation. This equation treats $N_{1}$ and $N_{2}$ equally and gives us very similar but slightly higher $p$-value.
} 

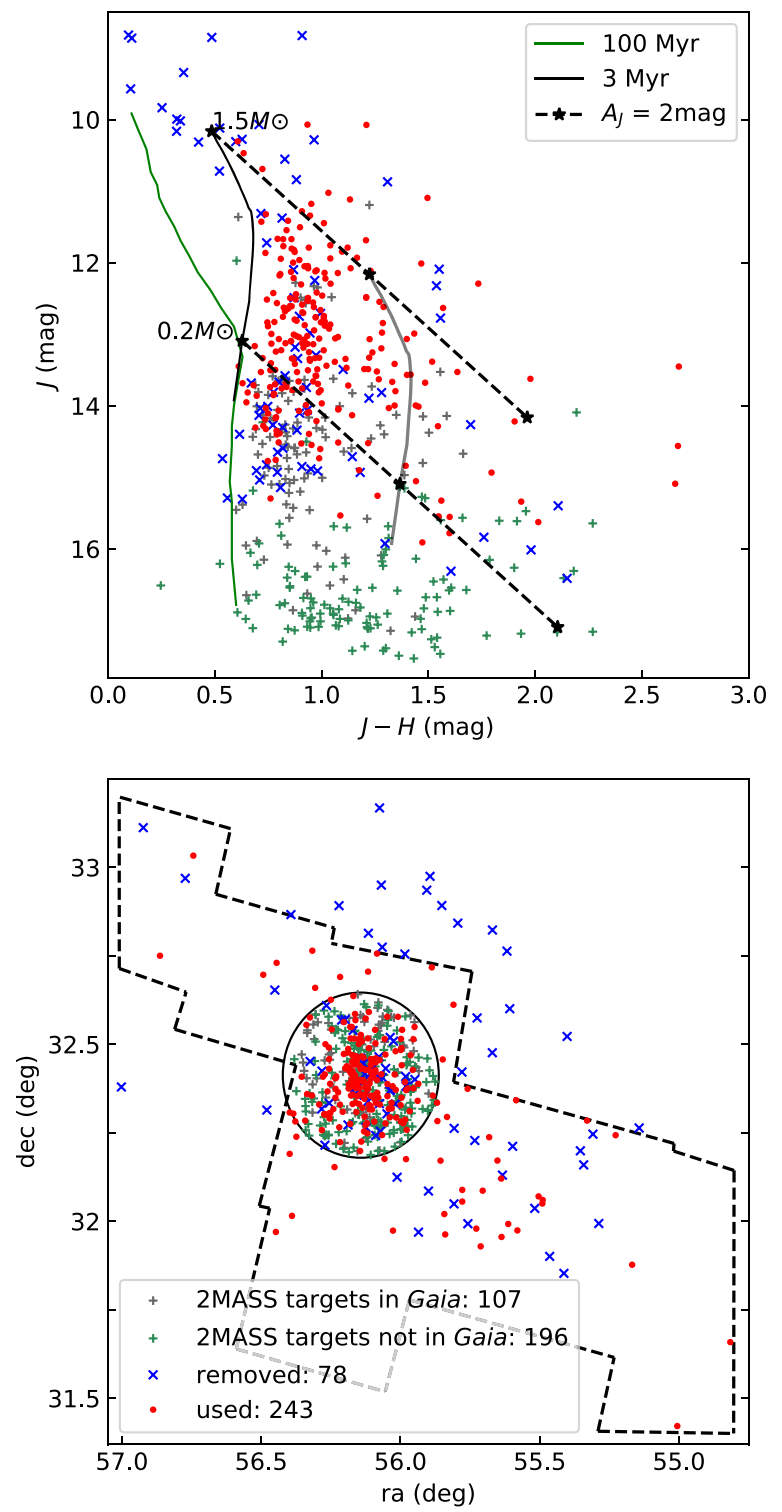

Figure 6. Upper panel: CMD of sources in IC 348. In our sample, 243 observed sources are shown as red dots, 78 observed sources not in our sample are shown as blue crosses. Other 2MASS sources within $14^{\prime}$ from the star B5 star HD 281159 are marked as gray and green pluses (see text). An extinction vector of $A_{J}=2 \mathrm{mag}$ is shown as the length between two black asterisks on the dashed black line. Lower panel: spatial distribution of these sources. The dashed black line shows the IRAC field of view.

sources with Gaia data, 107 satisfy the cut of proper motion (no more than 6 mas $\mathrm{yr}^{-1}$ away from the cluster median) and distance (no more than $200 \mathrm{pc}$ away from the cluster median). They are shown as gray pluses in Figure 6, and the 196 2MASS sources without Gaia data are marked with green pluses.

The resulting $p$-value of the $2 \mathrm{D} \mathrm{K-S}$ test is shown in Figure 7. The extinction limit of $A_{J}$ is chosen to vary from 2 to 6 since the highest extinction in this cluster $\left(A_{J}=5.5\right)$ is no greater than 6 . By inspection of Figure 7 , we conclude that our sample is representative for $0.25-1.5 M_{\odot}$ at $A_{J}=6$ in IC 348 .

\subsubsection{Orion A}

In Figure 8, the 1431 red points are stars in our final sample of Orion A and the 406 blue pluses are considered to be cluster members, but are removed from analysis. Red and blue add up to

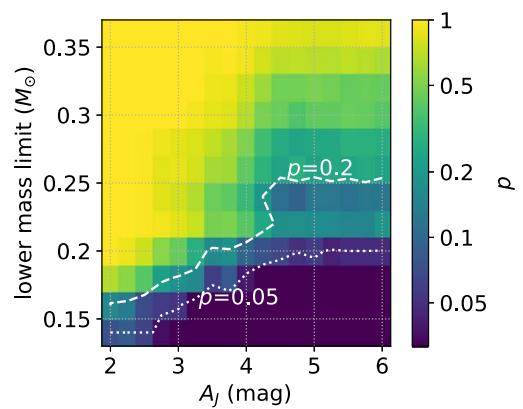

Figure 7. K-S test significance level $(p)$ for the null hypothesis that the extinction-limited, mass-limited IC 348 sample is uniformly drawn from the total. See Figure 5.

the 1837 stars retained in Section 2.2.1. Since the Orion structure extends over a large spatial area, it is relatively difficult to selected unobserved cluster members from 2MASS sources. First of all, including all other 2MASS sources located within the large structure may introduce many contaminants in the total. Hence, we only take the 8650 sources in the FoV of the densest region $\left(-6^{\circ}<\right.$ decl. $<-4.5,83^{\circ} .25<$ R.A. $\left.<84.25\right)$ into consideration. The 599 non-member (candidates) identified by Da Rio et al. (2016) are excluded to remove contaminants. Among the 8650 sources, 5238 have Gaia data, with 1505 satisfying the astrometry criteria for cluster members applied in Sections 2.3.1 and 2.3.2. They are shown as gray pluses in Figure 8. The 3412 sources not in Gaia are shown as green pluses.

Another potential caveat is that the representative mass range and extinction limit of our Orion A sample may depend on radius (the distance between individual stars from the cluster center), e.g., due to the presence of differential extinction. In the right panel of Figure 8 , three circles with radii of $5^{\prime}(0.58 \mathrm{pc}), 15^{\prime}(1.73 \mathrm{pc})$, and $30^{\prime}$ (3.46 pc) from $\Theta^{1}$ Orionis $\mathrm{C}$ (marked as the yellow asterisk) are drawn. We show a zoom-in of the region within $15^{\prime}$ from the central star. It can be seen that our sample is less complete in the crowded center. This inhomogeneity can also be verified in Figure 9, where a stacked histogram of sources as a function of radius is shown. The colors have the same meaning as in Figure 8. From a radial distance of $1^{\prime}$ to $5^{\prime}$, the ratio of the number of stars in our sample (red) to the total (red+blue+gray+green) is just $\sim 7 \%$, while from $5^{\prime}$ to $30^{\prime}$, this value increases to $\sim 19 \%$.

Hillenbrand et al. (1998) has found that the optically thick disk fraction in ONC increases toward the cluster center. If the representative mass range of our sample is a function of radius, then this could be a systematic effect for the estimated disk frequencies. Therefore, we assess the representativeness of our Orion A sample by separating them into different radial bins, and individually applying the $2 \mathrm{D} \mathrm{K}-\mathrm{S}$ test (assuming an age of $2 \mathrm{Myr}$ and a distance of $396.7 \mathrm{pc}$ ). The results are presented in Figure 10. In each panel, the red cross lying on the $p=0.2$ contour marks the lower mass limit at the extinction limit of $A_{J}=3.0$. It is evident from this figure that the significance level $(p)$ returned by the test increases as we go to the outer radius, indicating that our sample is less representative in the inner part. Therefore, we exclude 88 red points within $5^{\prime}$ from $\Theta^{1}$ Orionis $C$ from our sample, and conclude that the new sample with $1343(=1431-88)$ YSOs is representative from 0.34 to $1.5 M_{\odot}$ at the extinction limit of $A_{J}=3.0$, which is the location of the red cross in the upper right panel of Figure 10.

By inspection of the left panel of Figure 8, we can see that at the extinction limit of $A_{J}=3.0$, there are a few stars more 

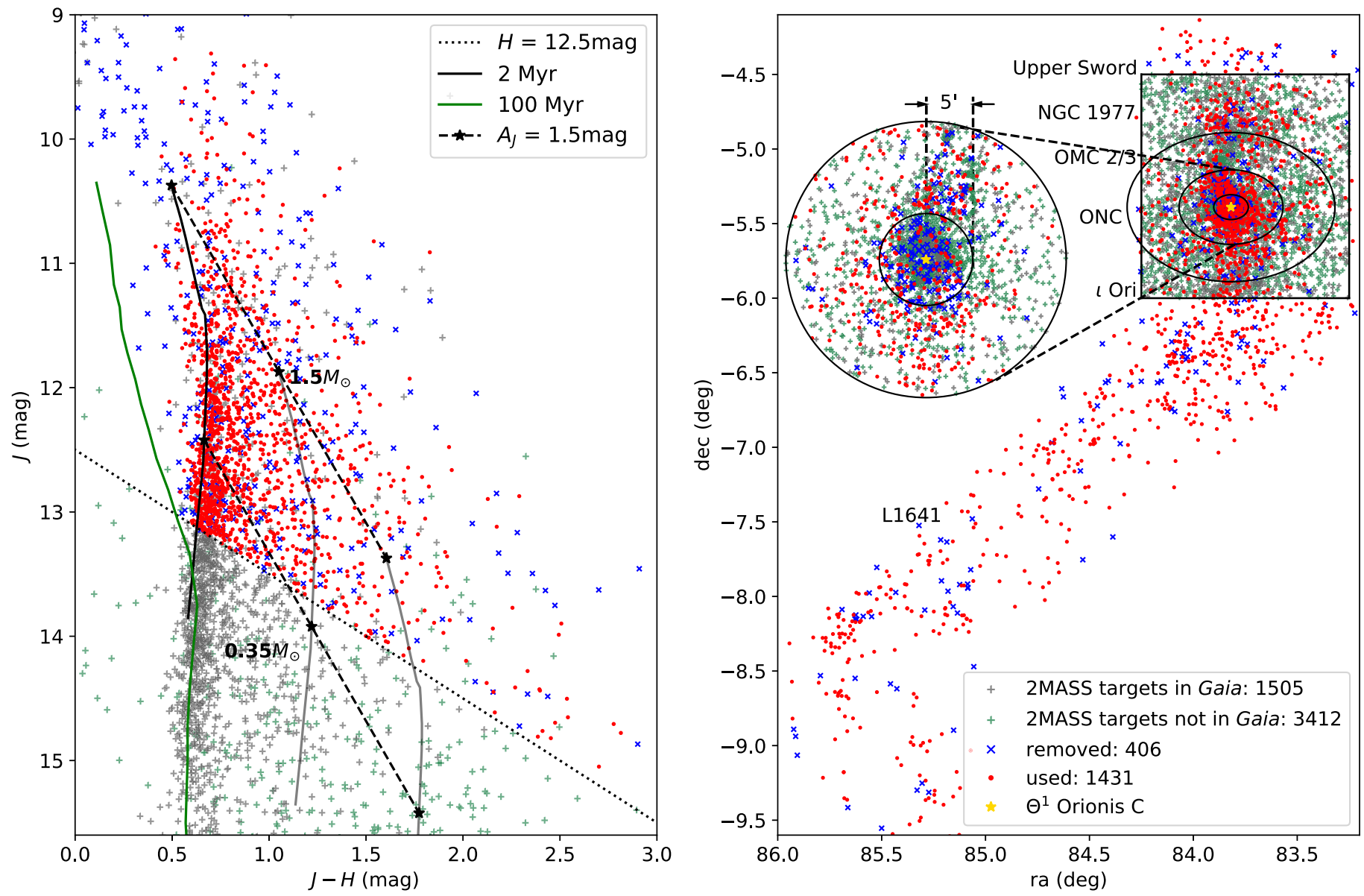

Figure 8. Left panel: CMD of sources in Orion A. In the final sample, 1431 observed members are shown as red dots, and 406 observed members not in the final sample are shown as blue crosses. Other 2MASS sources not observed are marked as gray and green pluses (see text). An extinction vector of $A_{J}=1.5$ is shown as the length between two asterisks on the dashed black line. Right panel: spatial distribution of these sources. The black square marks the field containing the Orion Nebula, NGC 1977, and OMC-2/3 (following Figure 14 of Megeath et al. 2012). The yellow asterisk marks the location of $\Theta^{1}$ Orionis C, which is an O6 star at the center of ONC.

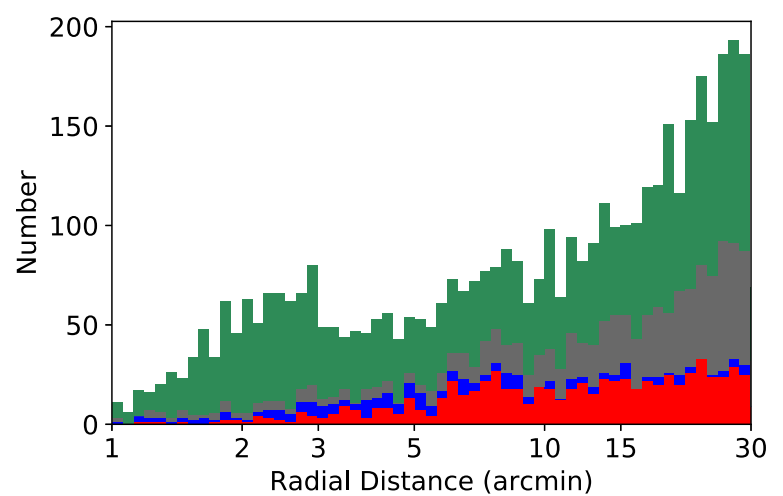

Figure 9. Number distribution as a function of radial distance from $\Theta^{1}$ Orionis $\mathrm{C}$ of cluster members in our sample (red), cluster members removed from our sample (blue), 2MASS sources with Gaia data that survive the cuts on proper motion and distance (gray), and 2MASS sources not in the Gaia catalog (green). Only the range of radial distance from $1^{\prime}$ to $30^{\prime}$ is shown. The $X$-axis is in the logarithm scale.

massive than $0.35 M_{\odot}$ falling below the $H=12.5$ detection limit. This may indicate that our method used to assess representativeness is, for some reason, yielding too low (high) limits to stellar mass (extinction). We can check if the selection is indeed representative by calculating the cumulative distribution function (CDF) of stellar masses for the samples in each region, both the "used" ones (red) and the whole distributions (red+blue+gray+green, in Figures 4, 6, and 8. Note that in Orion A we have omitted all targets within $5^{\prime}$ from the center). At the $A_{J}$ limit and representative mass range of each cluster, we infer the extinction-corrected $J$-band magnitude by dereddening along the extinction vector on the $J-(J-H)$ panel, and convert $M_{J}$ to stellar mass using the BCAH98 isochrones. The resulting CDFs are shown in Figure 11, along with the number of sources in each sample $\left(N_{1}, N_{2}\right)$. We perform a onedimensional K-S test for the null hypothesis that the two samples are drawn from the same continuous distribution. The returning $p$-value is quite high in all regions.

Therefore, the lower mass limits selected by the $p=0.2$ contours in Figures 5, 7, and 10 are still considered to be acceptable. In Section 4, we will only calculate disk frequencies in the representative mass ranges using stars within the $A_{J}$ limits. In Section 4.4 where disk fractions in Orion A are studied, we will also investigate if the results change a lot by adopting a more restrictive extinction limit of $A_{J}<1$.

\subsection{Discussion of This Assessment}

\subsubsection{Distance Dispersion in Each Cluster}

In the above assessment, we assume a median cluster distance for all stars in each cluster. However, in Table 1, we 


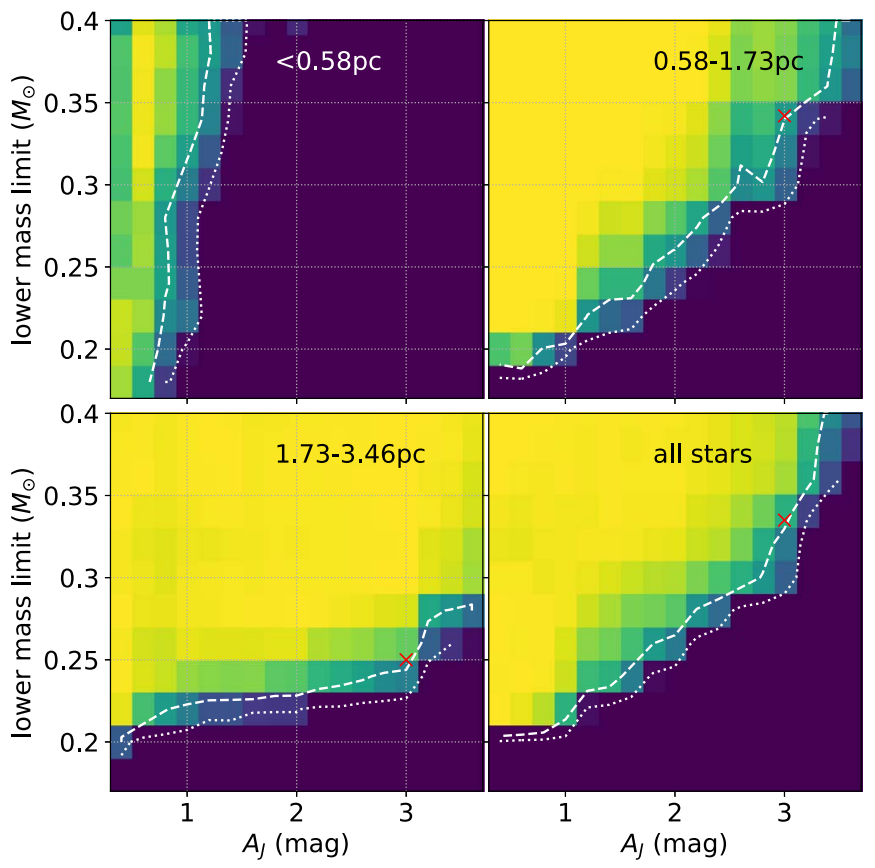

Figure 10. K-S test significance level $(p)$ for the null hypothesis that our extinction-limited, mass-limited Orion A sample is uniformly drawn from the total. The upper left panel considers all sources within $5^{\prime}(0.58 \mathrm{pc})$ from $\Theta^{1}$ Orionis C. The upper right and lower left panels consider sources from $5^{\prime}$ to $15^{\prime}$ and from $15^{\prime}$ to $30^{\prime}$ from $\Theta^{1}$ Orionis $C$, respectively (see the right panel of Figure 8 for the location of the two annuli). The lower right panel considers all sources shown in Figure 8. The dashed and dotted lines mark the contours of $p=0.2$ and $p=0.05$, respectively. The scale of the color bar is the same as in Figures 5 and 7.

notice that the inter-quartile range compared to the median distance is already large. In NGC 1333, IC 348, and Orion A, this value is $(329.3-282.3) / 300.7=15.6 \%,(343.0-304.2) /$ $324.2=12.0 \%$, and $(415.1-380.0) / 396.7=8.8 \%$, respectively. In Table 1, we consider effects from such a distance dispersion by quoting the calculated representative mass range using the 25 th percentile and 75 th percentile distances at the same extinction limit. The lower mass limits in IC 348 and Orion A are raised by $0.02 M_{\odot}$ if adopting a restrictive distance estimation (75th percentile).

\subsubsection{The Usage of $2 M A S S$ Sources}

We are aware of the fact that the whole population of each cluster is not red+blue + gray+green, but how far is the total of real cluster members different from our assumption? Since sources marked with red, blue, and gray have already passed the membership criteria of Gaia proper motion and distance, the fraction of contamination for them should be very low. Thence, below we only focus on discussing the 2MASS sources shown as green pluses, for which astrometric data are not available. Note that in Figures 4, 6, and 8, the number of gray (green) pluses with inferred stellar mass in the corresponding representative mass ranges and extinction limits are 4 (7), 19 (7), and 119 (32), respectively. Therefore, although there is a substantial amount of green pluses, only a small fraction of them are inside of the region where $2 \mathrm{D} \mathrm{K}-\mathrm{S}$ tests are performed.

We need to take two questions into consideration: (1) How many stars not in the clusters are included in the green? (Note that green pluses stand for 2MASS sources not included in the

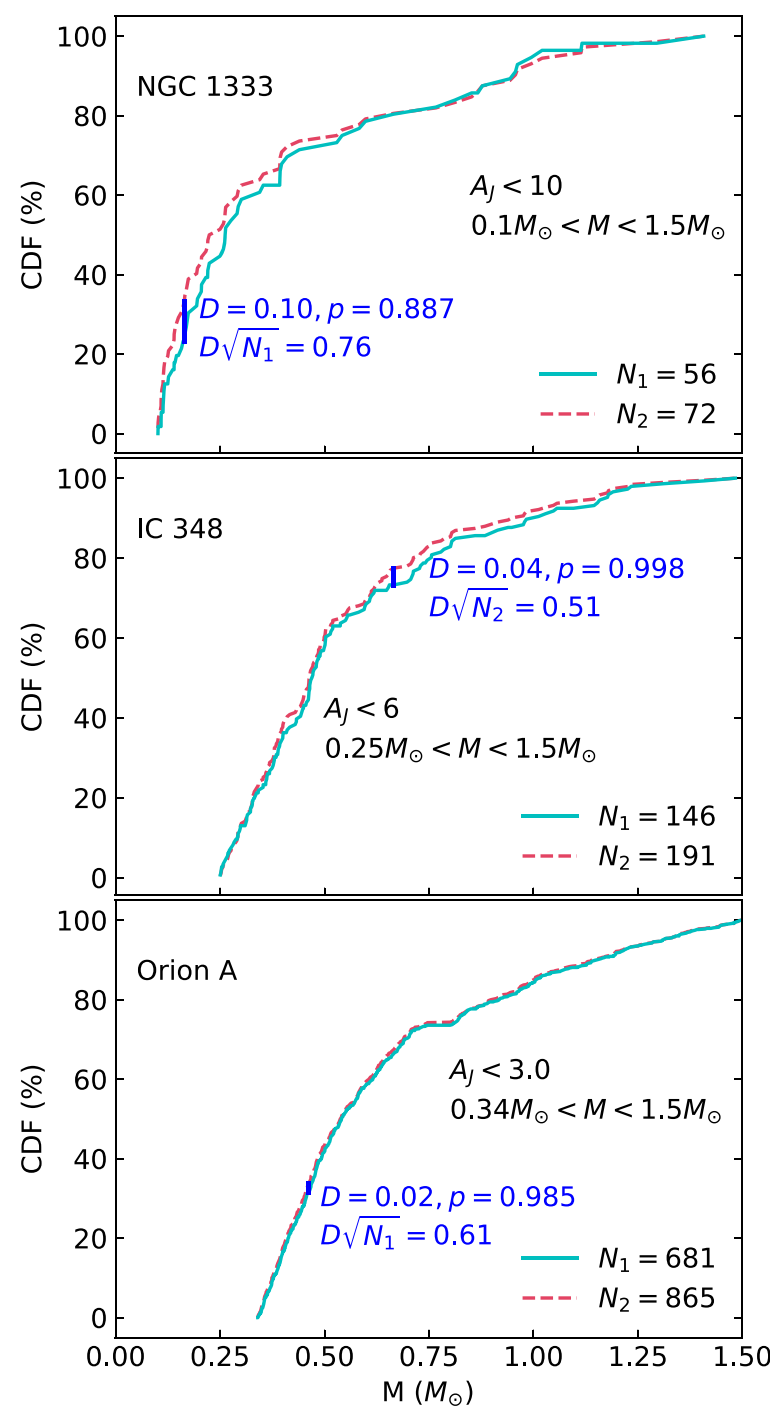

Figure 11. Cumulative distribution function (CDF) of stellar masses for the samples in each region within the representative mass ranges and $A_{J}$ limits. In each panel, the dashed pink line shows CDF of the whole population, while the solid cyan line shows CDF of the used stars. The blue line segment indicates the maximum distance between two CDFs.

Gaia catalog.) (2) How many stars in the clusters are not included in the green? To answer the first question, we need to consider contaminations from both background stars and foreground field stars. It is hard to include background stars in the representative mass range of the green because they are old, distant, and reddened by the cloud. It is easy to imagine that on each $J-(J-H)$ plane, they generally lie on the left side of the cluster isochrone, or on the right side of the isochrone but below our adopted lower mass limit.

Foreground stars are closer to us but suffer from little extinction, so most of them lie upward of (brighter than) the $1.5 M_{\odot}$ dashed boundary, where no green plus resides. Lowmass foreground stars fall easier into the region where K-S tests are performed. However, using the Wainscoat et al. (1992) Galactic star count model, Wilking et al. (2004) estimated a small number of Galactic field stars in the densest region of NGC $1333(\sim 10 \%)$. Since these authors performed a deeper near-infrared survey $(H \leqslant 16.5)$ than ours $(H \lesssim 13)$, the contamination of IN-SYNC should be less compared to $10 \%$. This is probably also true for IC 348. In Orion A, the 
contamination can be potentially higher because of the relative lower extinction of this cluster. However, since many sources brighter than $H=12.5$ have been observed, and most field stars (candidates) are removed in Section 2.2.1, we also have reasons to believe that the contamination is also a small fraction.

The second question is comparable to asking if any cluster members in the representative mass range are fainter than the 2MASS limit. The answer is probably true, in the sense that protostars with high extinction are harder to detect. However, we do not think these sources in our sample for which we classified the spectra are actually protostars. That is very unlikely. However, they have such unusual infrared colors that we are not confident of the extinction estimates, and therefore not confident of the disk properties. They clearly have significant circumstellar material, probably dominated by larger envelope emission, but they are rare in our sample and we prefer to remove them (to some extent making our IR excess fractions lower limits). The removal of protostars is presented in Section 3.4.

Therefore, although the assessment in Section 2.3 is not perfect, we still consider it to be an acceptable way to determine the representative mass range of an extinctionlimited sample.

\subsubsection{Is There a Bias toward Disk-bearing YSOs?}

We have demonstrated that the finally used sample in each cluster is not biased as a function of location on the $J-(J-H)$ CMD. Since disk properties are crucial to this work, however, another consideration is that stars with disks are easier to identify by previous membership studies. Therefore, it is possible that the used sample is biased toward sources with mid-IR excesses. We check if this is indeed the case by considering the cumulative disk frequencies more massive than a certain stellar mass in the representative mass range and extinction limit. The results from the "used" sample and the total are shown as the solid blue and dashed pink line in Figure 12, respectively. The background shades indicate the $1 \sigma$ poisson statistical uncertainty $\left(\sqrt{N_{\text {disk }}} / N_{\text {all }}\right)$. Please see Section 3.2 and 3.3 for the definition of "disk frequency" in this paper. In principle, the number of sources in the two samples in Figure 12 should be the same as in Figure 11. However, since some unused stars do not have IRAC data, and are thus excluded from the calculation, fewer sources are used in creating Figure 12.

Statistically speaking, the cumulative disk frequency as a function of stellar mass for the "used" sample is consistent with the total, in the sense that the differences are all less than $1 \sigma$. Therefore, we conclude that our samples are also representative in terms of disk properties.

\section{The Disk Diagnostic: $4.5 \mu \mathrm{m}$ Excess}

Widely used diagnostics to distinguish between stars with and without disks include (but may not be limited to): (1) the amount of infrared continuum excess; (2) color-color plots constructed from combined 2MASS, WISE, and Spitzer photometry; (3) infrared slope of the spectral energy distribution (SED). Given that the effective temperature (spectral type) are known for all stars in our sample, we adopt the first approach as our primary disk diagnostic. In Section 3.1, we describe our IRAC IR data. The determination of extinctions
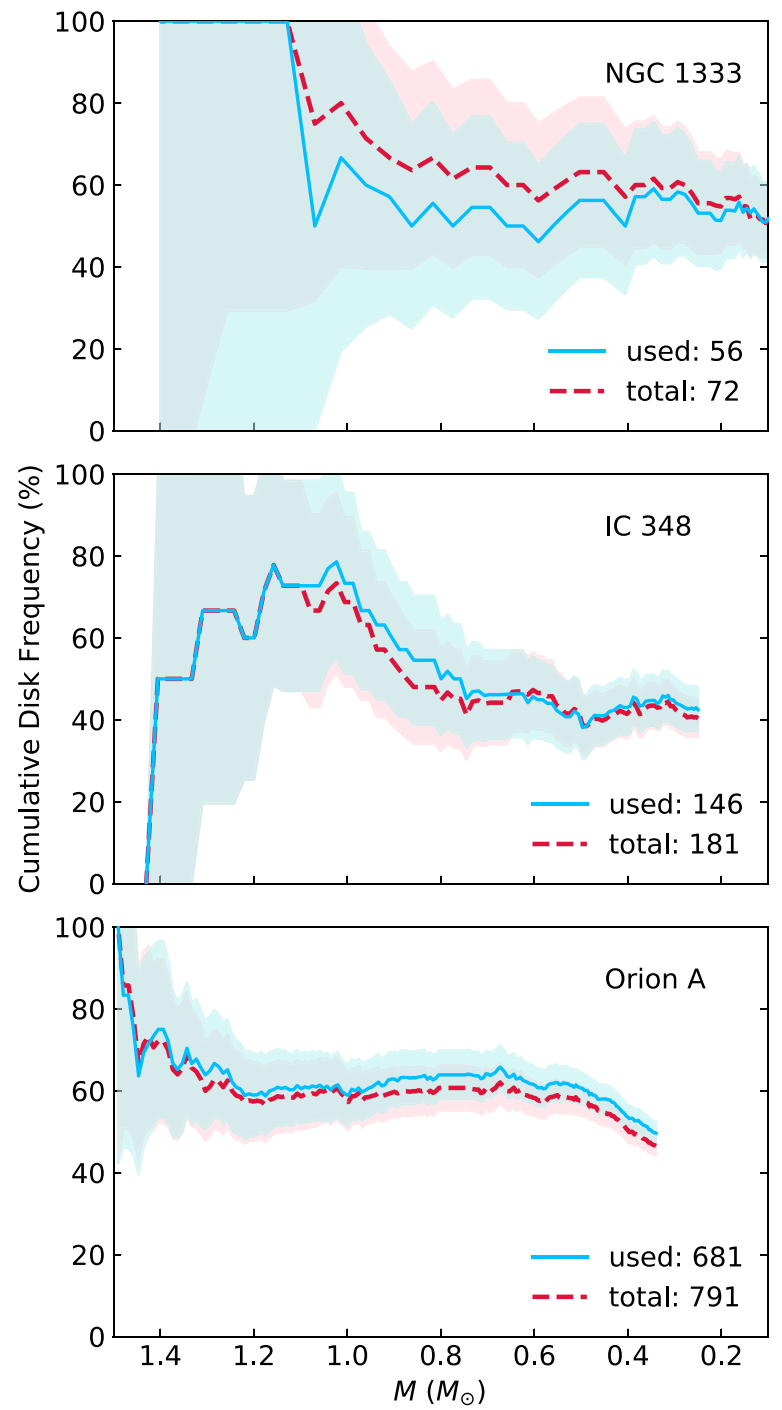

Figure 12. Cumulative disk frequency for the samples in each region within the representative mass ranges and $A_{J}$ limits. In each panel, the dashed pink line shows results of the whole population, while the solid blue line shows results of the used stars. The light pink and light cyan shades indicate statistical uncertainties.

and infrared excesses are outlined in Section 3.2. Our definition of the primordial disk is given in Section 3.3. The removal of protostars is described in Section 3.4.

\subsection{IRAC Data}

As stated above (see Section 2.2.3), our photometric data are reduced by two different programs that have used different reduction techniques. To determine if there is a systematic difference between the flux reported by SEIP and c2d catalogs, we collect $\sim 30,000$ sources in the Perseus region that have been reduced by both programs. We show the difference of their reported magnitudes as a function of the $\mathrm{c} 2 \mathrm{~d}$ magnitude in Figure 13. Magnitudes are converted from flux densities by adopting the zero magnitude flux given in the IRAC Instrument Handbook. Sources too bright or too faint are cut off to only consider the range of magnitudes covered by our IN-SYNC sample. Generally speaking, for the same sources, SEIP magnitudes are greater than that in $\mathrm{c} 2 \mathrm{~d}$, especially in the two channels at 5.8 and $8.0 \mu \mathrm{m}$. We perform an unweighted least- 
$[3.6]$
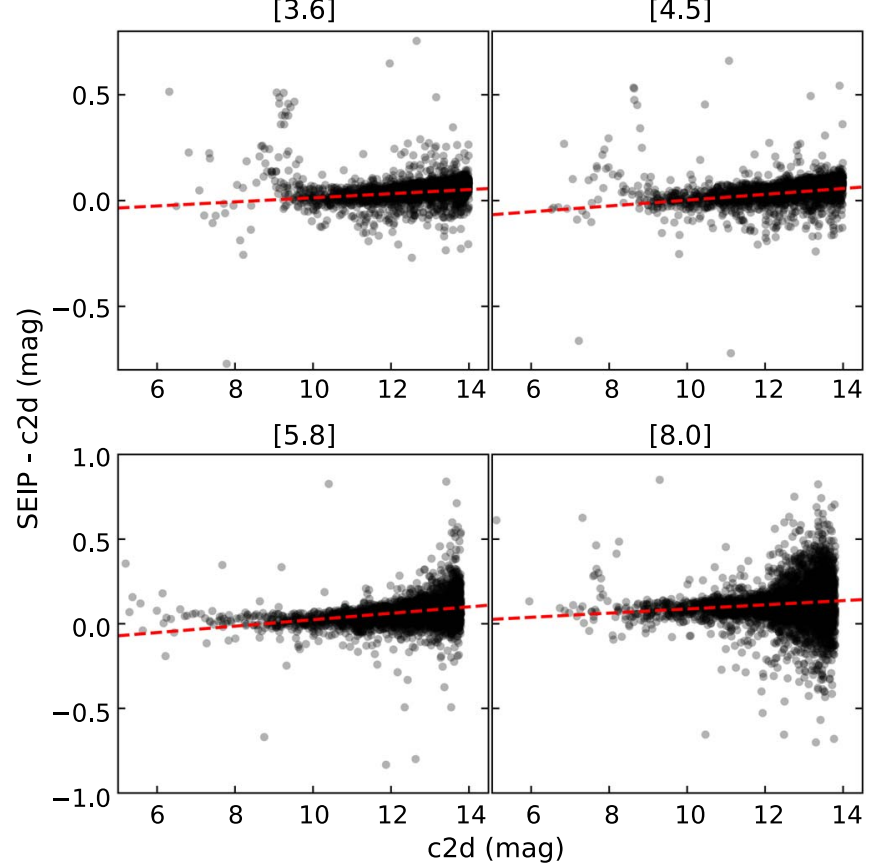

Figure 13. Difference of magnitudes reported by SEIP and c2d as a function of the $\mathrm{c} 2 \mathrm{~d}$ magnitude for a set of sources that have been reduced by both programs. The red lines show the fitted least-squares linear regression.

squares linear regression for the IRAC four channels, as shown as red lines in Figure 13. To mitigate uncertainties introduced by the inhomogeneity, we add this offset (red lines) from the c2d magnitudes for sources in the Perseus sample. Figure 13 also shows that there is not only a shift but also a scatter between the photometry of c $2 \mathrm{~d}$ and SEIP. Below we explain how photometric uncertainties reported by $\mathrm{c} 2 \mathrm{~d}$ and SEIP are inflated to match the observed scatter in Figure 13.

The IRAC photometric uncertainties reported by $\mathrm{c} 2 \mathrm{~d}$ are at least $0.05 \mathrm{mag}$, which is measured by the repeatability of flux measurements. Since this systematic error is not considered by SEIP, but is likely present in the data, we first add a floor of $0.05 \mathrm{mag}$ to the SEIP uncertainties. After that, SEIP still underestimates flux uncertainties compared with c2d. Therefore, before adding the systematic error of $0.05 \mathrm{mag}$, we multiply the original SEIP errors by a factor of 1.2 at 3.6 and $4.5 \mu \mathrm{m}$, a factor of 1.8 at $5.8 \mu \mathrm{m}$ and a factor of 2.0 at $8.0 \mu \mathrm{m}$ to bring it to the same scale with $\mathrm{c} 2 \mathrm{~d}$. We examine the trend of the scatter in Figure 13 as a function of magnitude by calculating a running rms residual with a window size of 1 mag and a step size of $0.1 \mathrm{mag}$. The resulting trend is shown as the black line in Figure 14. A floor of 0.01 and 0.02 mag are further added to $\sigma_{I_{3}}$ and $\sigma_{I_{4}}$ to bring flux uncertainties to at least the order of the observed scatter in Figure 13. The finally used scale of flux uncertainties is shown as the blue and red dots in Figure 14.

\subsection{Determination of Infrared Excess}

The color we observe for a YSO is a combination of effects from photospheric color, reddening, and intrinsic excesses. We follow the definition of intrinsic IR excess given by Hillenbrand et al. (1998):

$$
\Delta(J-H)=(J-H)_{0}-(J-H)_{\text {photo }}
$$

where $(J-H)_{0}=(J-H)_{\mathrm{obs}}-E(J-H)$ is the extinctioncorrected color, and $(J-H)_{\text {photo }}$ is the contribution of the underlying stellar photosphere. To estimate extinction, we assume $\Delta(J-H)=0$, use the extinction law for $R_{V}=3.1$ from Cardelli et al. (1989) integrated over the 2MASS filters from Cohen et al. (2003), and adopt $(J-H)_{\text {photo }}$ as a function of $T_{\text {eff }}$ from Pecaut \& Mamajek (2013).

The intrinsic infrared excess is expressed by $\Delta\left(K_{\mathrm{s}}-I_{\mathrm{x}}\right)=$ $\left(K_{\mathrm{S}}-I_{\mathrm{x}}\right)_{0}-\left(K_{\mathrm{S}}-I_{\mathrm{x}}\right)_{\text {photo }}$ (here $x=1,2,3,4$, standing for the four IRAC channels). With the values of $A_{J}$, we can easily calculate $\left(K_{\mathrm{s}}-I_{\mathrm{x}}\right)_{0}=\left(K_{\mathrm{s}}-I_{\mathrm{x}}\right)_{\mathrm{obs}}-E\left(K_{\mathrm{s}}-I_{\mathrm{x}}\right)$ by adopting the extinction law at 3-8 $\mu \mathrm{m}$ derived by Flaherty et al. (2007).

To determine how $\left(K_{\mathrm{s}}-I_{\mathrm{x}}\right)_{\text {photo }}$ varies as a function of $T_{\mathrm{eff}}$, we calculate synthetic colors by convolving model spectra with the response curve of $2 \mathrm{MASS}$ and IRAC filters. Using the "BTSettl" synthetic spectra (Allard et al. 2012), we compute colors for solar metallicity, no $\alpha$-element enhancement models with $3.0<\log g<5.0$ and $3000 \mathrm{~K}<T_{\text {eff }}<5000 \mathrm{~K}$. All of the stars in our sample have $\log g$ and $T_{\text {eff }}$ measurements within these ranges. In the bottom four panels of Figure 15, the synthetic colors are shown as the dashed magenta lines, solid orange lines, and dash-dotted red lines for $\log g=3.0,4.0$, and 5.0 , respectively. The red asterisks, blue dots, and cyan crosses are extinction-corrected colors of sources in our sample. We notice that there appears to be a tight locus of these data points, which should represent emission dominated by stellar photospheres. While the models describe the largescale structure of the diskless loci reasonably well, there are discrepancies at the $\sim 0.05 \mathrm{mag}$ level. To eliminate these, we derive the $\left(K_{\mathrm{S}}-I_{\mathrm{x}}\right)_{\text {photo }}-T_{\text {eff }}$ relation by empirically fitting the observed loci.

To select a number of sources along the loci for fitting we follow three steps for each $\left(K_{\mathrm{s}}-I_{\mathrm{x}}\right)_{0}-T_{\text {eff }}$ panel: (1) We utilize the $K$-means clustering algorithm (Lloyd 1982) to partition data into two groups, and retain the group with smaller $\left(K_{\mathrm{S}}-I_{\mathrm{x}}\right)_{0}$ mean. In Figure 15, we indicate the decision boundary by assigning a background color to each group (light yellow and light gray). This step allows us to remove most outliers that are sources with excesses. 1104, 1047, 947, and 844 sources are retained for the fitting of $\Delta\left(K_{\mathrm{s}}-I_{1}\right), \Delta\left(K_{\mathrm{s}}-I_{2}\right), \Delta\left(K_{\mathrm{s}}-I_{3}\right)$, and $\Delta\left(K_{\mathrm{s}}-I_{4}\right)$, respectively. Some sources only have IRAC data at shorter wavelengths due to a loss of sensitivity as we go to longer wavelengths. (2) For the remaining $\sim 1000$ sources, we iteratively fit a linear relation between $\left(K_{\mathrm{s}}-I_{\mathrm{x}}\right)_{0}$ and $T_{\text {eff }}$ with $3 \sigma$-clipping-any outliers more distant than three times the rms of the fit residuals are removed. (3) By eye inspection of data points on the locus, we expect the $\left(K_{\mathrm{s}}-I_{\mathrm{x}}\right)_{\text {photo }}$ color slightly increase with decreasing stellar temperatures. This should be a real feature because synthetic colors also show this characteristic. Therefore, we further iteratively fit a two-degree spline of the remaining data with $2.5 \sigma$-clipping. In order to prevent over-fitting, we manually set the interior knots at 3200 , 3600 , and $4100 \mathrm{~K} .973,867,750$, and 626 sources are finally used for the fitting of $\Delta\left(K_{\mathrm{s}}-I_{1}\right), \Delta\left(K_{\mathrm{s}}-I_{2}\right), \Delta\left(K_{\mathrm{s}}-I_{3}\right)$, and $\Delta\left(K_{\mathrm{s}}-I_{4}\right)$, respectively. These sources are shown in deeper colors in the corresponding panels.

We also need to know the uncertainty in fitting the locus of diskless stars. To this end, we consider all the sources with $\left(K_{\mathrm{s}}-I_{\mathrm{x}}\right)_{\text {obs }}<\left(K_{\mathrm{s}}-I_{\mathrm{x}}\right)_{\text {locus }}$, i.e., those data points lying below the black lines on the bottom panels in Figure 15. Then $\sigma_{\left(K_{\mathrm{s}}-I_{\mathrm{x}}\right)_{\text {locus }}}$ is evaluated by the rms of $\left(K_{\mathrm{s}}-I_{\mathrm{x}}\right)_{\text {locus }}-$ $\left(K_{\mathrm{s}}-I_{\mathrm{x}}\right)_{\mathrm{obs}}$ of these sources. We expect that the scatter of data 

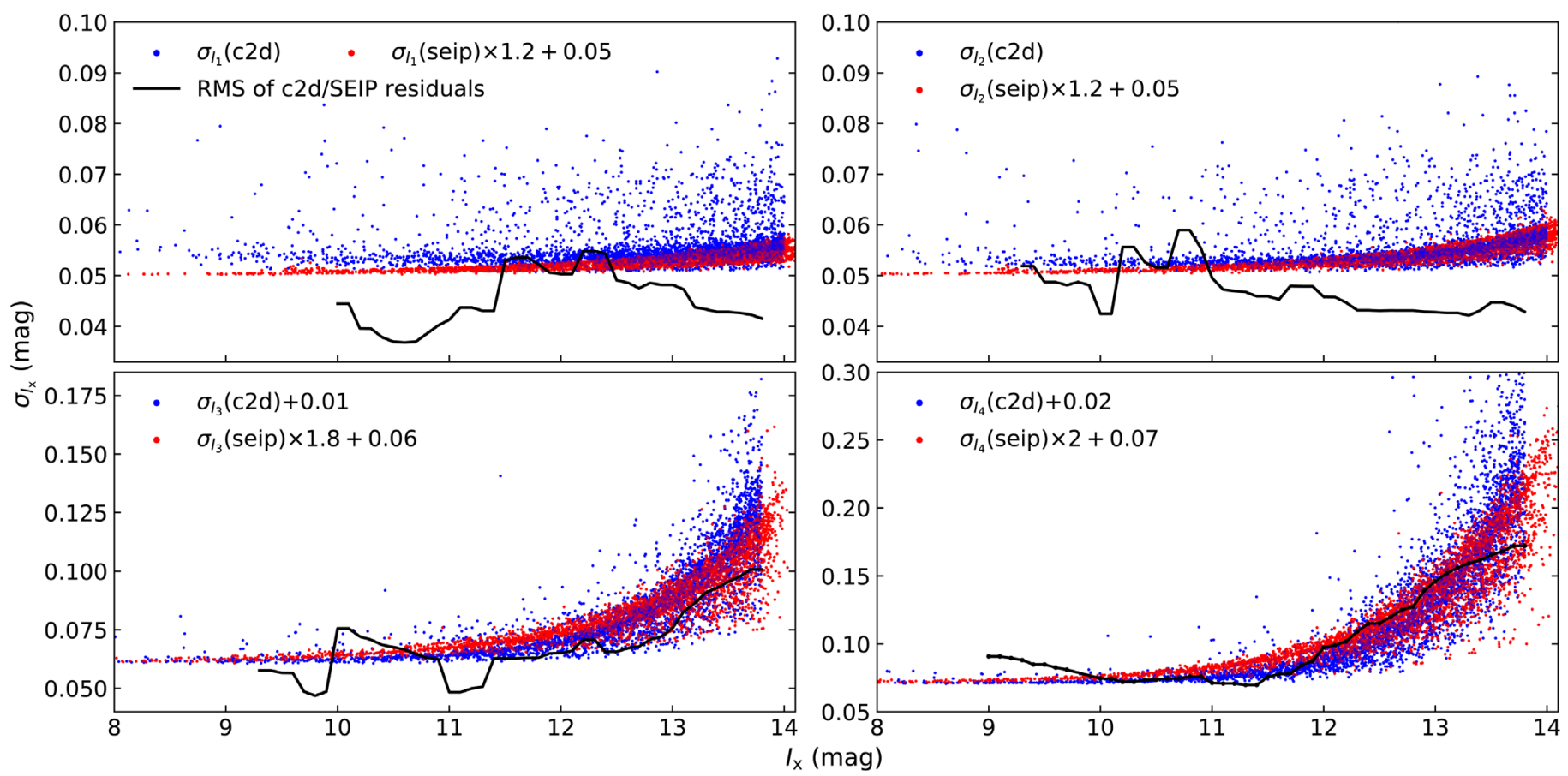

Figure 14. IRAC photometric uncertainties as a function of magnitude in the four channels. The black lines show the rms residuals of the observed scatter in Figure 13. Blue and red dots are data from the c2d and SEIP delivery, respectively. The legend in each panel shows how the original photometric uncertainties are increased to be at least in the same order of the black line.

points above the fitted lines should be very similar to that below the lines. The uncertainties are $0.060,0.078,0.153$, and $0.250 \mathrm{mag}$ at $3.6,4.5,5.8$, and $8.0 \mu \mathrm{m}$, respectively.

\subsection{Disk Classification}

Sources are thought to possess excess at a certain wavelength if the intrinsic color exceeds the fitted relation by both 3 times the scatter of our fitting $\left(\sigma_{\left(K_{s}-I_{x}\right)_{100 u s}}\right)$ and 3 times the internal uncertainty associated with the flux $\left(\sigma_{\left(K_{\mathrm{s}}-I_{x}\right)_{0}}\right)$, i.e.,

$$
\begin{aligned}
& \Delta\left(K_{\mathrm{s}}-I_{\mathrm{x}}\right)>3 \times \sigma_{\left(K_{\mathrm{s}}-I_{\mathrm{x}}\right)_{\text {locus }}} \\
& \Delta\left(K_{\mathrm{s}}-I_{\mathrm{x}}\right)>3 \times \sigma_{\left(K_{\mathrm{s}}-I_{\mathrm{x}}\right)_{0} .}
\end{aligned}
$$

A crucial issue in this method is that $3.6-8.0 \mu \mathrm{m}$ emission for a disk irradiated by a very faint star is coming from much smaller radii than if irradiated by a much brighter star. Therefore, there is an observational bias that it is harder to probe the excess for the faintest stars. To mitigate the effects from such a bias, we hope that even for the lowest-mass stars, the typical amount of $\Delta\left(K_{\mathrm{S}}-I_{\mathrm{x}}\right)$ should be much larger than both $3 \times \sigma_{\left(K_{\mathrm{s}}-I_{\mathrm{x}}\right)_{\text {locus }}}$ and $3 \times \sigma_{\left(K_{\mathrm{s}}-I_{\mathrm{x}}\right)_{0}}$. Is this really the case?

To answer this question we make use of disk SEDs computed by Robitaille et al. (2006). In Figure 16, we show in black lines the amount of excess that we expect to see if there are fiducial primordial disks around stars of four different masses. Fiducial disks are selected from a grid of SEDs by requiring age $=1-3 \mathrm{Myr}$, inner disk radius $=$ sublimation radius, etc. In colored lines we show typical values of max $\left(3 \times \sigma_{\left(K_{\mathrm{s}}-I_{x}\right)_{\text {locus }}}, 3 \times \sigma_{\left(K_{\mathrm{s}}-I_{X}\right)_{0}}\right)$ in the three clusters.

As demonstrated in Figure 16, the typical values of excess threshold, $\max \left(3 \times \sigma_{\left(K_{\mathrm{s}}-I_{x}\right)_{\text {locus }}}, 3 \times \sigma_{\left.\left(K_{\mathrm{s}}-I_{x}\right)_{0}\right)}\right.$, are as large as (and sometimes larger than) the amount of excess at 3.6 and $5.8 \mu \mathrm{m}$. However, at 4.5 and $8.0 \mu \mathrm{m}$, a fiducial disk should be selected by our criterion, since the amount of excess exceeds that of the threshold. In this paper, we define stars with primordial disks as those possessing excess at $4.5 \mu \mathrm{m}$.

\subsection{Removing Protostars}

The detection of infrared excesses can be attributed to the existence of either envelopes or disks. Protostars (flat spectrum, Class 0 or Class 1 sources) with dusty envelopes should be removed before the calculation of disk frequencies. Most experimental criteria used to identify protostars made use of IRAC 3.6, 4.5, and Multiband Imaging Photometer for Spitzer (MIPS) photometry at $24 \mu \mathrm{m}$. See Kryukova et al. (2012, their Equations (1) and (2)) for an example. However, since a few Perseus objects and most Orion sources don't have MIPS data in c2d or SEIP (because of bright nebulosity, saturation, and SEIP's strict cuts of extended sources), we use the following equation to remove protostars:

$$
\left(I_{1}-I_{2}\right)_{0}>0.7 \text {. }
$$

Envelope models predict that the above criterion (Allen et al. 2004) can separate most protostars from disk-bearing stars.

4 (5.4\% in NGC 1333), 4 (1.6\% in IC 348), and 11 (0.8\% in Orion A) sources are removed from the three clusters. After that, we are left with 70 sources in NGC 1333, 239 in IC 348, and 1332 in Orion A. A table of these sources is provided in the Appendix.

\section{Disk Frequency and Stellar Mass}

In this section we estimate the frequency of primordial disks for each cluster, and discuss evidence of trends in disk frequency over the representive mass range (Table 1). In Section 4.1, we compile a list of intermediate-mass stars in each region. The dependence of disk frequency on stellar mass is studied in Sections 4.2-4.4 for NGC 1333, IC 348, and Orion A, respectively. The results in the first two clusters are 

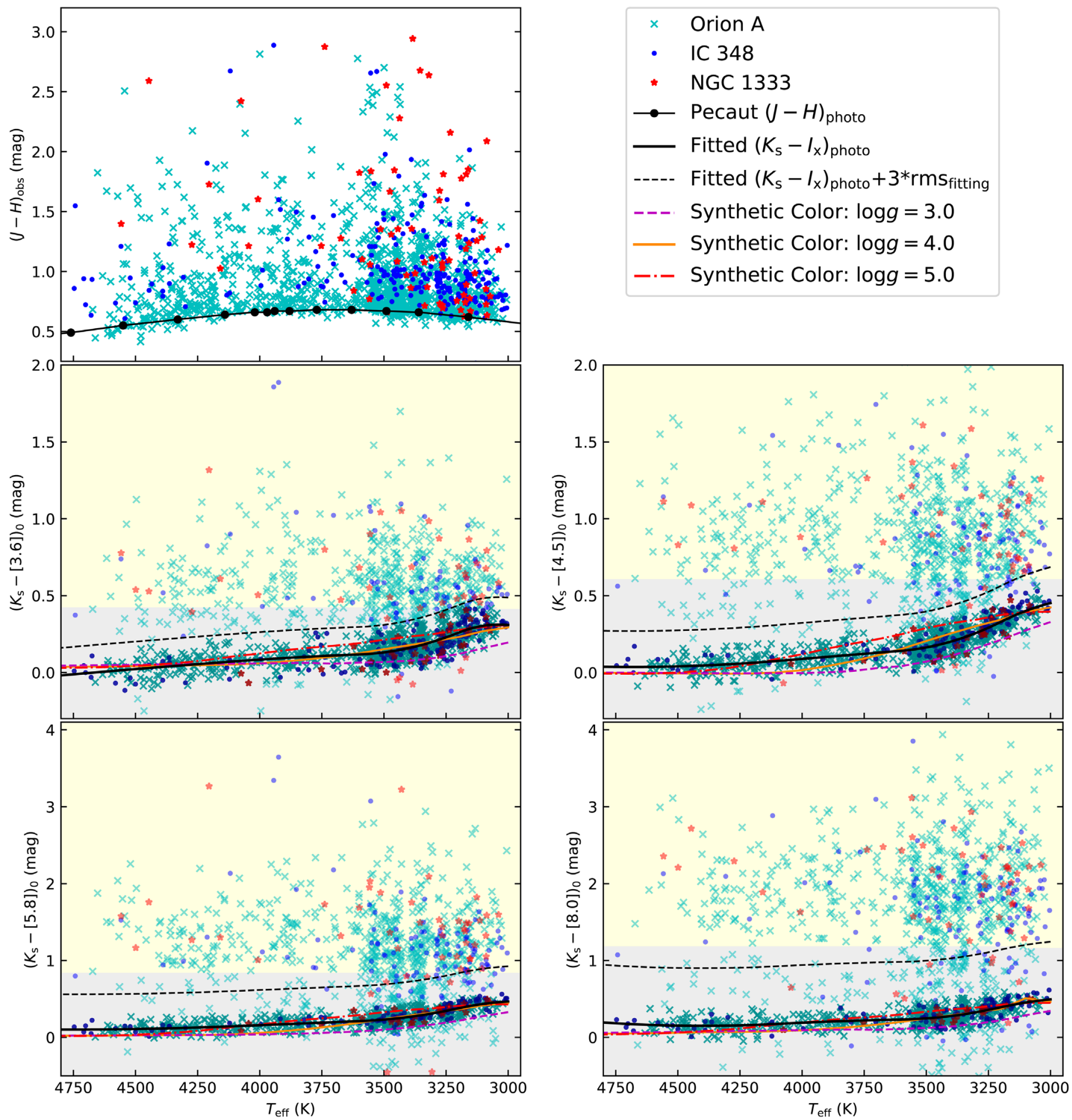

Figure 15. Upper left panel: observed $(J-H)$ as a function of $T_{\text {eff }}$. The other four panels: data points are extinction-corrected $\left(K_{s}-I_{\mathrm{x}}\right)_{0}$ color as a function as $T_{\mathrm{eff}}$ for sources in our sample (those in deeper colors are finally used to fit the color loci, see text); colored lines are synthetic colors calculated from spectra models; black lines are empirical colors derived by fitting the data. Dashed lines in the bottom four panels show $\left(K_{\mathrm{s}}-I_{\mathrm{x}}\right)_{\text {locus }}+3 \sigma_{\left(K_{\mathrm{s}}-I_{\mathrm{x}}\right)_{\text {locus }} \text {. }}$.

compared with the recent work of Luhman et al. (2016) in Section 4.5.

\subsection{Compiling a List of Intermediate-mass Stars}

To further compare the disk frequencies of our low-mass sample with that of higher-mass stars, we search from literature for intermediate-mass stars $\left(2.2-5 M_{\odot}\right)$. It is probable that focused membership studies can provide us with a complete sample of these early-type stars due to their brightness. The spectral types corresponding to this mass range in each cluster are outlined in Table 1 based on isochrone models by Siess et al. (20001; BCAH98 did not compute isochrones for $M>1.5 M_{\odot}$ stars).

We obtain 4 and 28 intermediate-mass stars in NGC 1333 and IC 348 from Luhman et al. (2016), all of which have c2d 


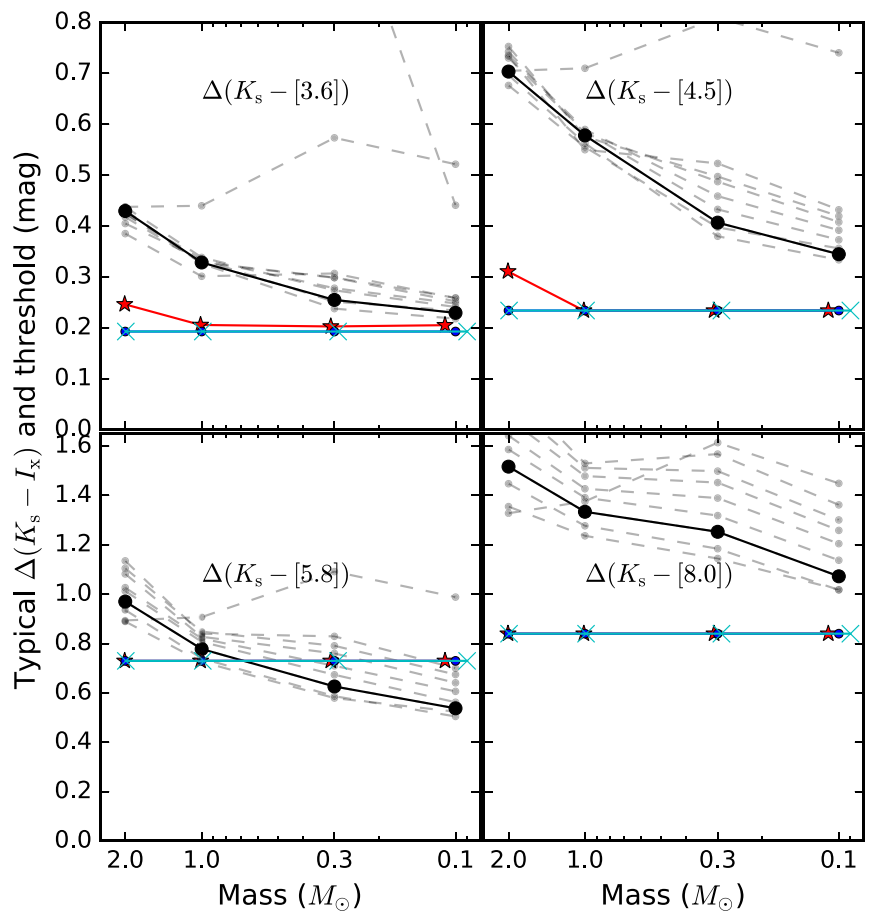

Figure 16. Black: expected colors of $\Delta\left(K_{s}-I_{x}\right)$ if there are fiducial disks around young stars with stellar masses of $2.0,1.0,0.3$, and $0.1 M_{\odot}$ (solid lines: viewed at an inclination of $63^{\circ} .26$; dashed lines: viewed at other inclinations from 18.19 to $\left.87^{\circ} .13\right)$. Colored lines: typical values of $\max \left(3 \times \sigma_{\left(K_{\mathrm{S}}-I_{\mathrm{X}}\right)_{\text {locus }} \text {, }}\right.$, $3 \times \sigma_{\left(K_{\mathrm{s}}-I_{\mathrm{x}}\right)_{0}}$ in NGC 1333, IC 348, and Orion A are marked in red asterisks, blue dots, and cyan crosses.

Spitzer/IRAC photometry except for the G0-type star 2MASS $03443200+3211439$ in IC 348 . We also remove the A3-type star SSTc2d J034432.0+321144 in IC 348 for its lack of 2MASS photometry. For Orion A, we obtain 55 intermediatemass stars in ONC from Da Rio et al. (2012), and 74 in L1641 from Hsu et al. (2012, 2013). Of them, $89(29+60)$ have extracted SEIP IRAC fluxes at $4.5 \mu \mathrm{m}$.

Extinction for these intermediate-mass stars are estimated in the same way as outlined in Section 3.2, but using the temperature (spectral type) scale for main sequence stars from Pecaut \& Mamajek (2013). Their photospheric colors $\left(K_{\mathrm{s}}-I_{\mathrm{x}}\right)_{\text {photo }}$ are assumed to be the same as a $1.5 M_{\odot}$ star. It is evident from Figure 15 and synthetic photometry that the loci do not vary greatly for stars more massive than $1.5 M_{\odot}$. Of the 89 stars in Orion A exhibit $\left(I_{1}-I_{2}\right)_{0}>0.7,2$ and are removed as protostars.

Now there are 4, 26, and 87 intermediate-mass stars in NGC 1333, IC 348, and Orion A, respectively. Similar to what we have done in the compilation of our low-mass star sample, we also apply the same proper motion and distance constraints outlined in Section 2.2.1 on these intermediate-mass stars, if Gaia data is available. After that, we are left with 4, 24, and 65 intermediate-mass stars in NGC 1333, IC 348, and Orion A.

\section{2. $N G C 1333$}

Assuming an average age of $1 \mathrm{Myr}$ for NGC 1333, we convert $T_{\text {eff }}$ into mass using the BCAH98 isochrone. After that, we separate our sample into two mass bins so that each bin contains 35 sources. Figure 17 demonstrates the derived disk frequency versus stellar mass. The blue line shows cumulative disk frequency as defined by the fraction of disks for all stars with stellar mass higher than a certain value. We have too few

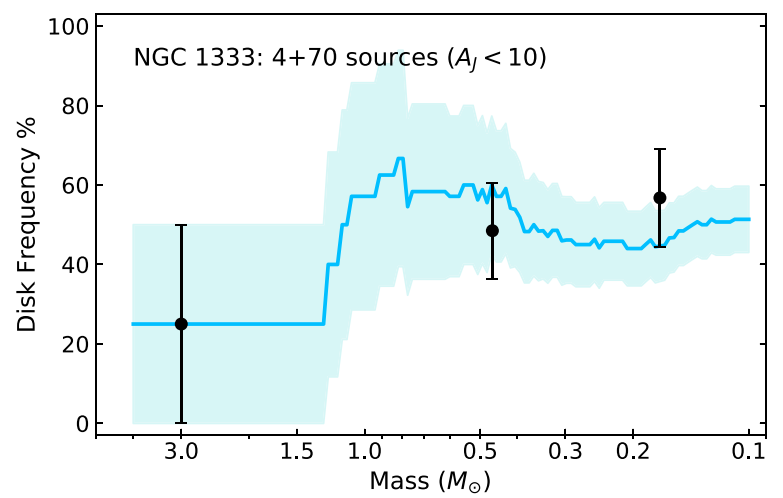

Figure 17. Black: disk frequency as a function of stellar mass in NGC 1333. Blue line: cumulative disk frequency derived by considering all stars more massive than a given mass ( $X$-axis).

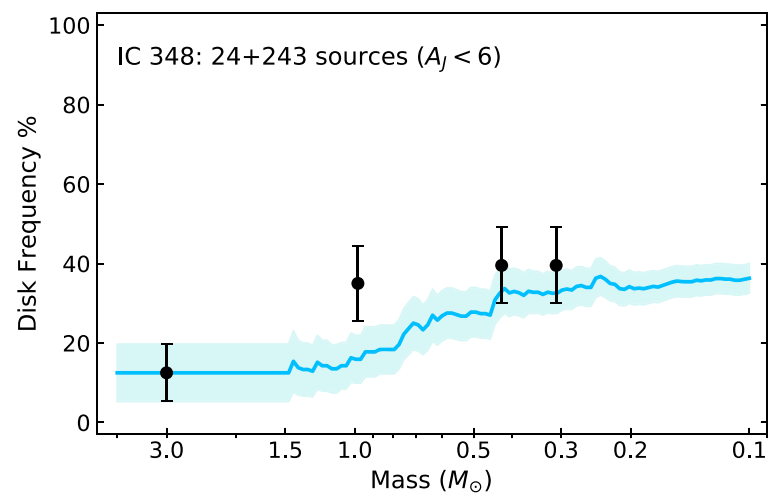

Figure 18. Disk frequency as a function of stellar mass in IC 348. We only bin stars more massive than $0.25 M_{\odot}$. See Figure 17 .

stars in the intermediate-mass range to allow robust comparison with low-mass stars. In the low-mass regime, our result is consistent with no mass dependence of disk fraction.

The derived overall disk frequency $\left(0.1-1.5 M_{\odot}, 52.9 \pm\right.$ $8.7 \%)$ is much lower than the $82.8 \pm 9.8 \%(72 / 87)$ given in Gutermuth et al. $\left(2008, M>0.08 M_{\odot}, A_{K_{\mathrm{S}}}<2\right)$. The latter disk frequency is examined by taking the ratio of Spitzer-identified YSOs with $K_{\mathrm{s}}<14$ in the "Main" cluster region over all 2MASS sources down to $K_{\mathrm{s}}=14$ after correcting for field-star contamination, which means that disk frequency is calculated as $\mathrm{N}(0 / \mathrm{I}+\mathrm{II}) / \mathrm{N}(0 / \mathrm{I}+\mathrm{II}+\mathrm{III})$. Therefore, the higher disk frequency in NGC 1333 reported by Gutermuth et al. (2008) can be caused by the facts that (1) they count protostars, which are removed from our analysis; and (2) their magnitude limit of $K_{\mathrm{s}}<14$ can be biased against diskless stars, since disk emission can already be prominent at $2 \mu \mathrm{m}$ under circumstances of low inclination angle, high accretion rate, flared disk geometry, or small inner disk hole size (Hillenbrand et al. 1998).

\subsection{IC 348}

Figure 18 shows disk frequency versus stellar mass in IC 348. The lowest disk frequency is in the highest mass bin $\left(2.2-5.0 M_{\odot}\right)$. As we go to lower-mass stars there is an increase of disk frequency. For low-mass stars $\left(0.25-1.5 M_{\odot}\right)$, disk frequency is consistent with little dependence on stellar mass.

Lada06 also analyzed a sample of $\sim 300$ known members in IC 348, and found four contiguous drops of disk fraction as one went to lower-mass stars from $1.0 M_{\odot}$ to $0.1 M_{\odot}$. It is worth checking why such a definite decline is not seen in our data. To 

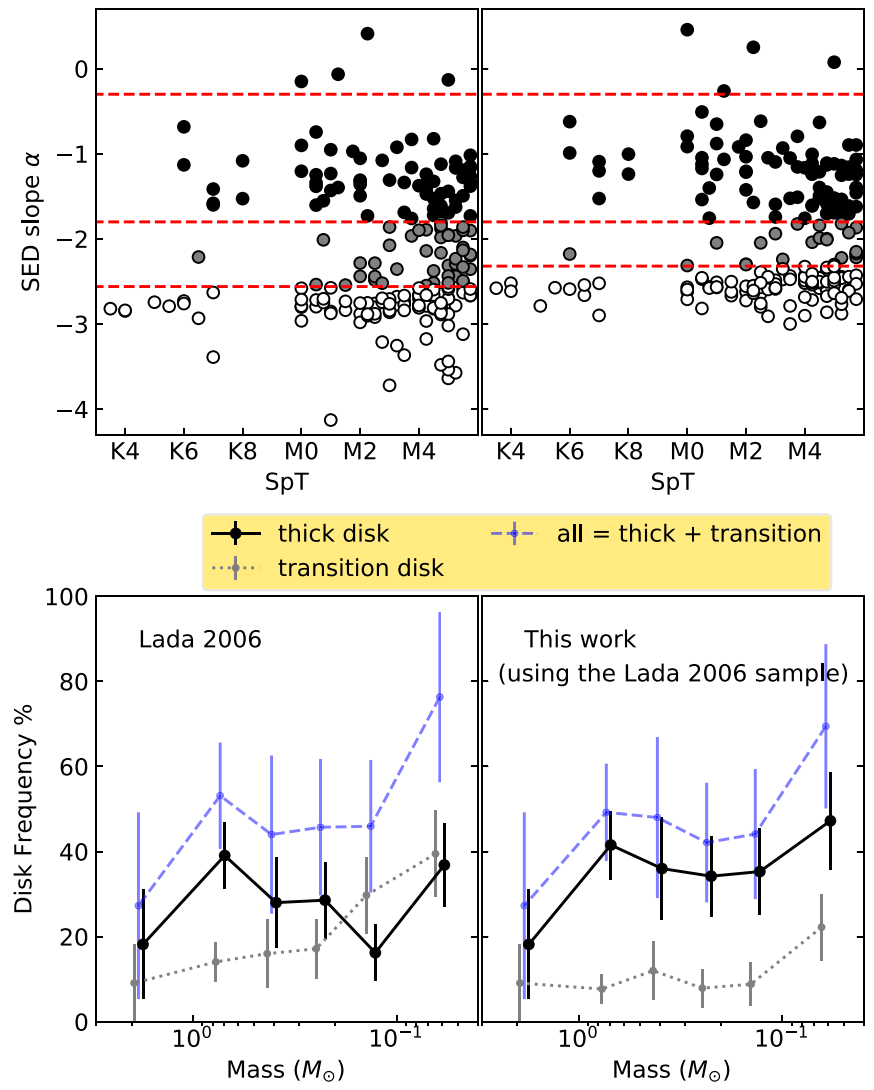

Figure 19. Upper left: $3.6-8.0 \mu \mathrm{m}$ de-reddened SED slope $(\alpha)$ determined by Lada06. $\alpha$ is defined in the $\lambda f_{\lambda}$ unit (Equation (4)). Dashed red lines $(\alpha=-2.56,-1.8,-0.3)$ show the separation of different disk properties adopted by Lada06. Upper right: $3.6-8.0 \mu \mathrm{m}$ de-reddened SED slope $\alpha$ as a function of spectral type. $\alpha$ are determined using $\mathrm{c} 2 \mathrm{~d}$ data and new estimation of extinction (Section 3.2). Thick, transition, and diskless stars are color-coded in black, gray, and white, respectively. Dashed red lines are $\alpha=-2.32,-1.8$, -0.3 . Bottom left \& Bottom right: Disk frequency as a function of stellar mass.

this end, we start with the 234 stars with spectral type between K3 and M6 in Lada06. For 214 stars that have data from the Spitzer GTO program at all IRAC bands, we show their dereddened SED slope measured by Lada06 as a function of spectral type in the upper left panel of Figure 19, and the resulting disk frequency in the bottom left panel of Figure 19. The spectral index (SED slope) is defined by

$$
\alpha=\frac{d \lg \left(\lambda f_{\lambda}\right)}{d \lg (\lambda)}
$$

and is measured by a simple power-law, least-squares fit to the four IRAC bands.

In the above analysis, spectral types, which are originally from Luhman et al. (2003), are converted into effective temperatures by fitting an APOGEE- $T_{\text {eff }}-S p T$ spline function using a set of 166 stars that have also been observed by INSYNC. The fitted relation is shown as the red line in Figure 20. $T_{\text {eff }}$ is then converted to stellar mass using the BCAH98 $3 \mathrm{Myr}$ model. Four protostars are removed by cutting sources with $\alpha>-0.3$ from analysis (Greene et al. 1994; Young et al. 2015). By and large this is just a repetition of the work of Lada06 with minor adjustments.

Apart from the different adopted disk diagnostics (this work: $4.5 \mu \mathrm{m}$ excess; Lada06: spectral index $\alpha$ ), their IRAC data is from the Spitzer GTO program, which is not exactly the same

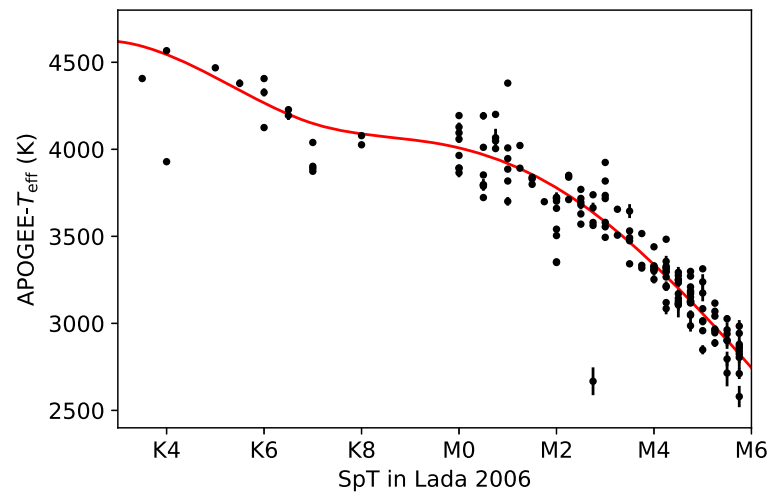

Figure 20. $T_{\text {eff }}$ measured from APOGEE spectra as a function of spectral type used in Lada06. Only 166 stars with $\sigma_{T_{\text {eff }}}<80 \mathrm{~K}$ are used in the fitting.

as being published by c2d (different reduction procedures can be a reason). We noticed that there are several diskless stars having extremely steep slopes $(\alpha<-3)$, whose magnitude at $8.0 \mu \mathrm{m}$ used by Lada06 can be more than $\sim 1$ mag fainter than that reported by $\mathrm{c} 2 \mathrm{~d}$. The photometric uncertainties reported by c2d are generally smaller at $8.0 \mu \mathrm{m}$. Furthermore, Lada06 used different methodology to estimate extinction, which may produce some systematic differences. In general, most stars in Lada06 have $A_{J}$ lower than our estimates.

These issues motivate us to redetermine extinction for the 234 stars with $\mathrm{K} 3<\mathrm{SpT}<\mathrm{M} 6$ by the method outlined in Section 3.2, and refit the de-reddened 3.6-8.0 $\mu \mathrm{m}$ SED slope for 213 stars that have c2d data at all IRAC bands. The newly determined $\alpha$ is shown as a function of spectral type in the upper right panel of Figure 19.

Compared with the upper left panel of Figure 19, where there is not a very distinct separation at $\alpha=-2.56$ between diskless stars and transition disks, ${ }^{10}$ the distribution of $\alpha$ for Class III stars determined by this work becomes tighter. We follow Lada06 by classifying all sources with $\alpha>-1.8$ as primordial disks, but change the maximum $\alpha$ for Class III objects to be -2.32 . The resulting disk frequencies are shown in the lower right panel of Figure 19. The general trend of our new disk frequencies is not sensitive to the new cutoff of $\alpha=-2.32$. A lot of sources termed as stars with "transition disks" are classified as stars with optically thick disks in our re-analysis. Hernández et al. (2007) also noted that the number of transition disks in Lada06 could be overestimated for the lowest-mass stars.

In conclusion, both Figure 18 and the lower right panel of Figure 19 demonstrate that in IC 348, primordial disk frequency is lower around sources more massive than $1 M_{\odot}$. But we do not observe a significant drop of disk frequency at $0.25-0.4 M_{\odot}$. This is also consistent with the result of Luhman et al. (2008).

\subsection{Orion A}

The upper panel of Figure 21 shows primordial disk frequency versus stellar mass in Orion $\mathrm{A}$. $T_{\text {eff }}$ is converted to stellar mass using the BCAH98 2 Myr model. Results for the lowest-mass stars are not shown since our sample is not representative for $M<0.36 M_{\odot}$ (Table 1, we adopt a restrictive estimate of the lower mass limit). The upper panel considers the $63+1373$ (intermediate-mass + low-mass)

\footnotetext{
${ }^{10}$ The transition disk is termed as the "anemic" disk in Lada06.
} 

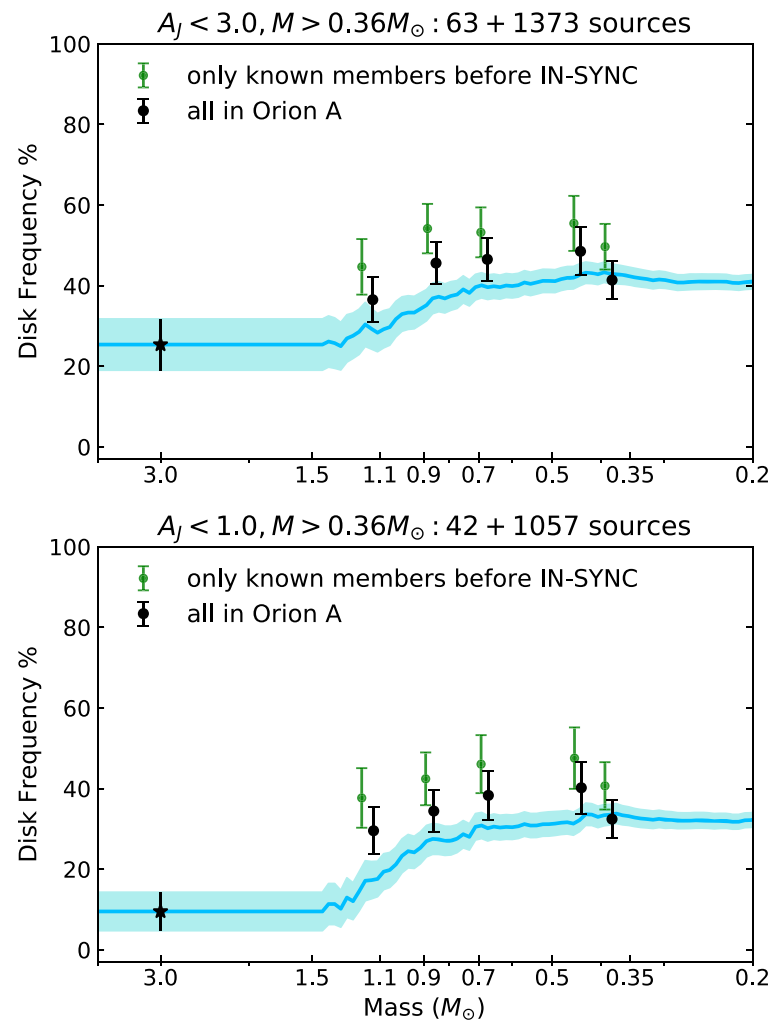

Figure 21. Disk frequencies as a function of stellar mass in the Orion A molecular cluster. We only bin stars more massive than $0.36 M_{\odot}$. The green data points present the results if only considering known members before the IN-SYNC study, which confirmed many non-disk-bearing stars. The upper panel considers 1373 low-mass sources and 63 intermediate-mass sources with $A_{J}<3.0$, while the lower panel only takes the $1057+42$ sources with $A_{J}<1.0$ into consideration. See Figure 17.

sources with $A_{J}<3.0$. From $M \approx 5 M_{\odot}$ to $M \approx 0.9 M_{\odot}$, there is an evident increase of disk frequency, which is consistent with the notion that disks live longer around lower-mass stars. In the mass bin of $0.36-0.42 M_{\odot}$, we observe a $6.5 \%$ drop of disk frequency compared with the bin of $0.42-0.5 M_{\odot}$. This decline is even more significant (beyond $1 \sigma$ uncertainty) if the lowest mass bin is chosen at $0.36-0.4 M_{\odot}$.

Disk frequencies in sub-regions in the Orion A molecular cloud have also been investigated by previous studies. Fang et al. (2013) diagnosed disk properties for $\sim 1000$ sources in L1641 using 2MASS-Spitzer SED slopes. Among three mass bins of $0.1-0.32 M_{\odot}, 0.32-1 M_{\odot}$, and $>1 M_{\odot}$, the highest disk frequency was found at $0.32-1 M_{\odot}$. Below we consider reasons that may produce the decline of the observed primordial disk frequency for the lowest-mass stars.

A straightforward explanation, as mentioned in Section 2.3.3, is that our method to assess representativeness underestimates (overestimates) the lower mass limit (extinction limit) for which our sample can be considered representative of the cluster population. Inspection of the left panel of Figure 8 reveals that at the extinction limit of $A_{J}=1.0$, no cluster members more massive than $0.35 M_{\odot}$ would drop below the detection limit of $H=12.5$. Therefore, we further study disk frequencies of stars with $A_{J}<1.0$, which gives us the lower panel of Figure 21. Disk frequencies in the lower panel are generally lower than that in the upper panel, because stars with primordial disks already have an excess at the $H$ band, which can render $\Delta(J-H)$ larger than 0 . Therefore, our estimation of $A_{J}$ for disk-bearing stars is larger

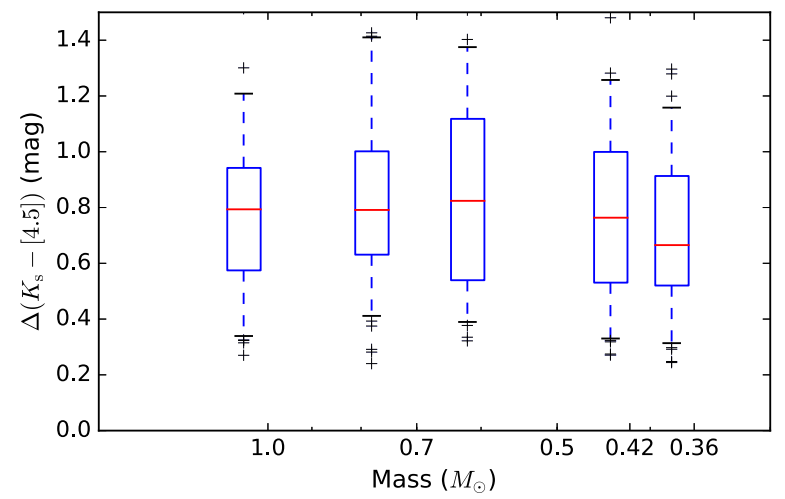

Figure 22. Box-and-whisker plot of $\Delta\left(K_{s}-[4.5]\right)$ for sources with disks in Orion $\mathrm{A}$ at the extinction limit of $A_{J}=3.0$. The box extends from the lower to upper quartile values, with a red line at the median. The whiskers extend from the box to show the range from 5th to 95th percentile of the data. Outliers are marked as plus symbols.

than the true value. By requiring $A_{J}<1.0$ we are excluding more disk-bearing stars than diskless stars. In the lower panel of Figure 21, the decline of primordial disk fraction at the lowest mass bin is still present. We conclude that the disk fractions we measure have not been biased to lower values by adopting an overly permissive extinction limit for our representative sample.

Another explanation is large degrees of dust settling (flattened geometry) or grain growth (D’Alessio et al. 2006; Hernández et al. 2007). Both effects will introduce an observational bias against the detection of gas-rich disks for low-mass stars. In Figure 22, we present the distribution of $4.5 \mu \mathrm{m}$ excess for sources identified as having disks by Equation (2). Indeed, the amount of excess decreases for stars in the mass range of $0.36-0.42 M_{\odot}$. Under scenarios of even the same degrees of dust settling or grain growth for stars of different masses, disks around low-mass stars are easier to drop below our detection limit given that the amount of fiducial excess for a $0.3 M_{\odot}$ star is already smaller than a $1 M_{\odot}$ star (see Figure 16). Therefore, we are unable to claim whether dust settling operates faster for low-mass stars, the question of which involves strong assumptions on disk structure and physics. However, if this is indeed the case, as suggested by Lada06 and Hernández et al. (2007), it could also be affecting NGC 1333 and IC 348 , even if no drop is apparent in the disk fractions.

\subsection{Comparison with Luhman et al. (2016)}

The recent census of NGC 1333 and IC 348 (Luhman et al. 2016) also investigates disk fractions as a function of spectral type. The authors collect Spitzer IR photometry from various literature and measure disk fractions as $N(\mathrm{II}) / N(\mathrm{II}+\mathrm{III})$. It is not explicitly specified whether the presence or absence of midIR excess is measured based on SED slope or excess colors. Their results (Figure 23 of Luhman et al. 2016) also show a higher disk fraction in NGC 1333, but their disk frequencies in both clusters are slightly higher than ours. The reason may originate from the fact that we have different definitions of "disk frequency." In this paper, we are only detecting optically thick inner disks with $4.5 \mu \mathrm{m}$ excess. For a few stars with only excess at $I_{3}$ or $I_{4}$, but not at $I_{1}$ or $I_{2}$, they are also counted as stars with disks in Luhman et al. (2016).

\section{Disk Frequency and Stellar Age}

In this section, we study a dependence of disk evolution-as probed by disk frequencies-as a function of stellar age. As an 

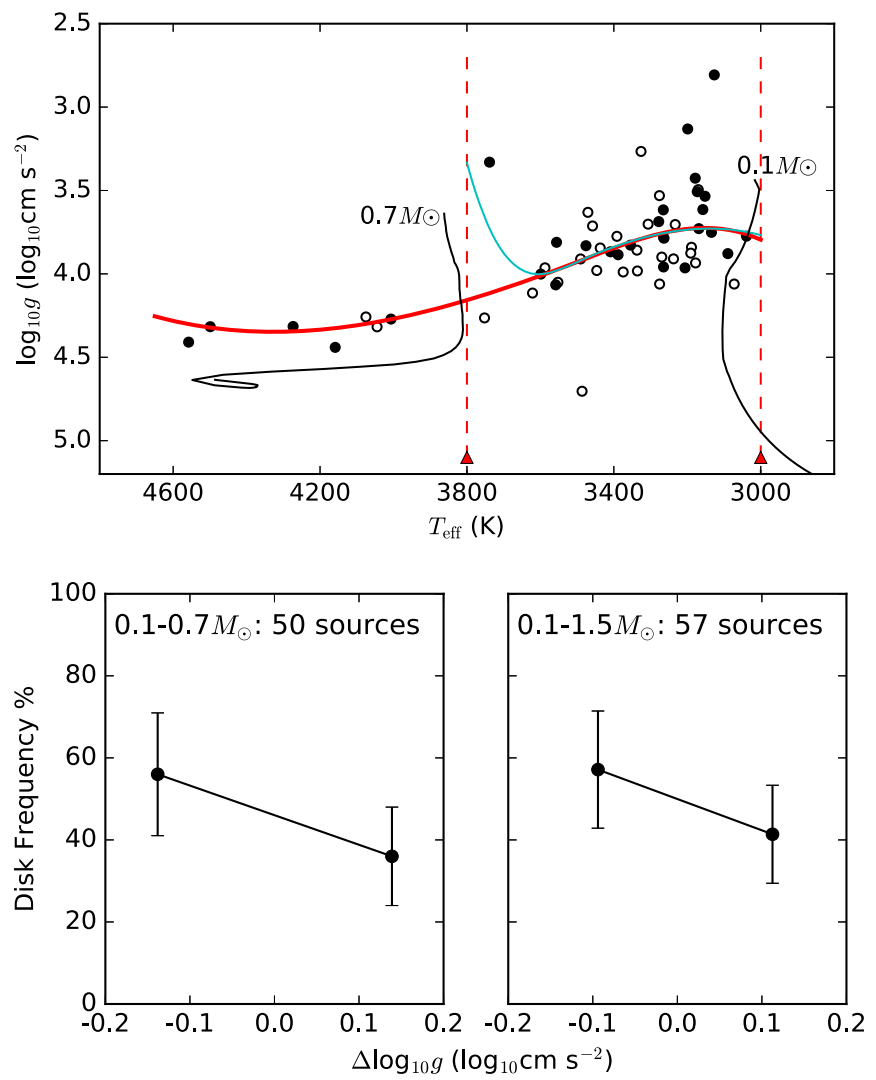

Figure 23. Upper panel: Distribution of spectroscopic $T_{\text {eff }}$ and $\log g$ in NGC 1333 (black dots for thick disks, and white dots for diskless stars). The red and cyan solid lines are median surface gravity derived by fitting cubic spline functions to the median-filtered trend of $\log g$ with $T_{\text {eff }}$ (red: using 57 stars with $4750 \mathrm{~K}<$ $T_{\text {eff }}<3000 \mathrm{~K}$; cyan: using 50 stars with $3000 \mathrm{~K}<T_{\text {eff }}<3800 \mathrm{~K}$ ). The black lines are BCAH98 evolutionary tracks for 0.1 and $0.7 M_{\odot}$ stars, and their corresponding effective temperatures are marked by the dashed red line. Bottom panel: Disk frequency as a function of $\Delta \log g$ (Equation (5)). In each mass range, the two bins of $\Delta \log g$ are divided to contain a similar number of sources.

age indicator, surface gravity should be independent of infrared excess (at least to the first order), and is thus independent of our classification of disk type. For this analysis, we further exclude any stars in our sample with $A_{J}$ higher than the extinction limit, $\sigma_{\log g}>0.2 \mathrm{dex}$, or $\sigma_{T_{\text {eff }}}>120 \mathrm{~K}$, leaving 57 sources in NGC 1333, 221 in IC 348, and 1200 in Orion A.

\subsection{NGC 1333}

The upper panel of Figure 23 shows the distribution of 57 sources in NGC 1333 on the $\log g-T_{\text {eff }}$ diagram. Disk-bearing stars are shown in black, and diskless stars are marked in white. We define

$$
\Delta \log g_{i}=\log g_{i}-\log g_{\text {median, } i},
$$

where $\log g_{i}$ is the surface gravity of the $i$ th star, and $\log g_{\text {median, } i}$ is the median surface gravity at the effective temperature of $T_{\text {eff, } i}$. We empirically determine $\log g_{\text {median }}$ by fitting a cubic spline to the median-filtered trend of $\log g$ with $T_{\text {eff }}$ using stars on the $\log g-T_{\text {eff }}$ plane. The red solid line is the resulting $\log g_{\text {median }}$ fitted by using all of the 57 stars, while the cyan line is $\log g_{\text {median }}$ fitted using the 50 YSOs between $3000 \mathrm{~K}$ and $3800 \mathrm{~K}$.

At a given (relatively low) effective temperature, a younger PMS star has been through shorter time of gravitational contraction along the Hayashi line (Hayashi 1961), and thus it
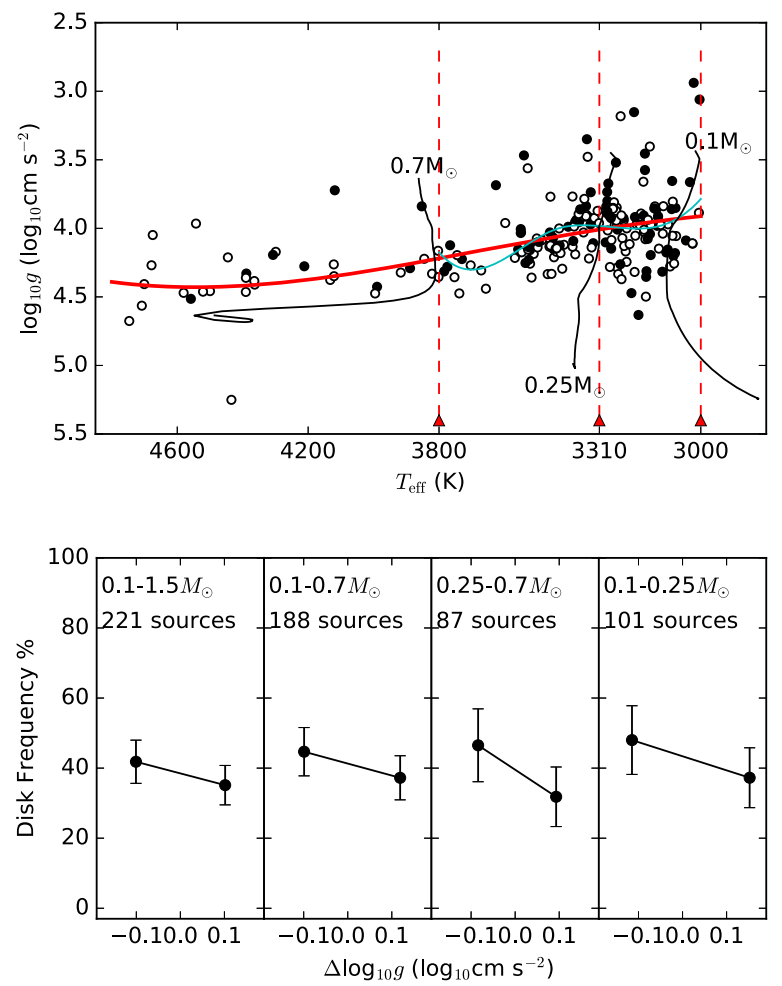

Figure 24. Upper panel: distribution of spectroscopic $T_{\text {eff }}$ and $\log g$ in IC 348 . Bottom panel: disk frequency as a function of $\Delta \log g$ (Equation (5)). See Figure 23.

typically has larger stellar radius and lower surface gravity, i.e., smaller $\Delta \log g$. However, if the effective temperature is relatively high (depending on stellar age and metallicity), a smaller $\Delta \log g$ may either indicate a younger age or a higher mass, because more massive stars $\left(M>0.7 M_{\odot}\right)$ will not remain convective at later stages of PMS evolution and would evolve toward higher $T_{\text {eff. }}$ The evolutionary tracks of a $0.1 M_{\odot}$ star and a $0.7 M_{\odot}$ star from the BCAH98 model are also shown in the upper panel of Figure 23. Their effective temperatures roughly correspond to $3000 \mathrm{~K}$ and $3800 \mathrm{~K}$, which are found by looking at the intersections of the evolutionary tracks and the red line. To investigate how disk frequency is dependent on stellar age, we first consider the 50 stars with 3000 $\mathrm{K}<T_{\text {eff }}<3800 \mathrm{~K},\left(0.1-0.7 M_{\odot}\right)$, because $\Delta \log g$ is directly related to stellar age in this temperature (mass) range. After that, we also look at the result from all of the 57 stars $(0.1-1.5$ $\left.M_{\odot}\right)$ to see if the trend of disk frequency changes. We adopt the red line as the median of surface gravity in all cases, since the cyan line overfits the data at the boundary region $(\sim 3800 \mathrm{~K})$.

The lower panel of Figure 23 shows disk frequency as a function of $\Delta \log g$. The two bins of $\Delta \log g$ are divided to contain the same number of sources. In each case, there is a decrease of primordial disk frequency for older stars. Including the seven sources more massive than $0.7 M_{\odot}$ does not change the general trend. Unfortunately, we do not have enough sources in this region to derive a strong conclusion that such a decrease in disk frequency indicates an evolution of primordial disks in NGC 1333.

\subsection{IC 348}

The upper panel of Figure 24 shows the distribution of sources in IC 348 on the $\log g-T_{\text {eff }}$ diagram. Overplotted are the BCAH98 evolutionary tracks of $0.1,0.25$, and $0.7 M_{\odot}$ stars. The median surface gravity is fitted in the same way as in the 


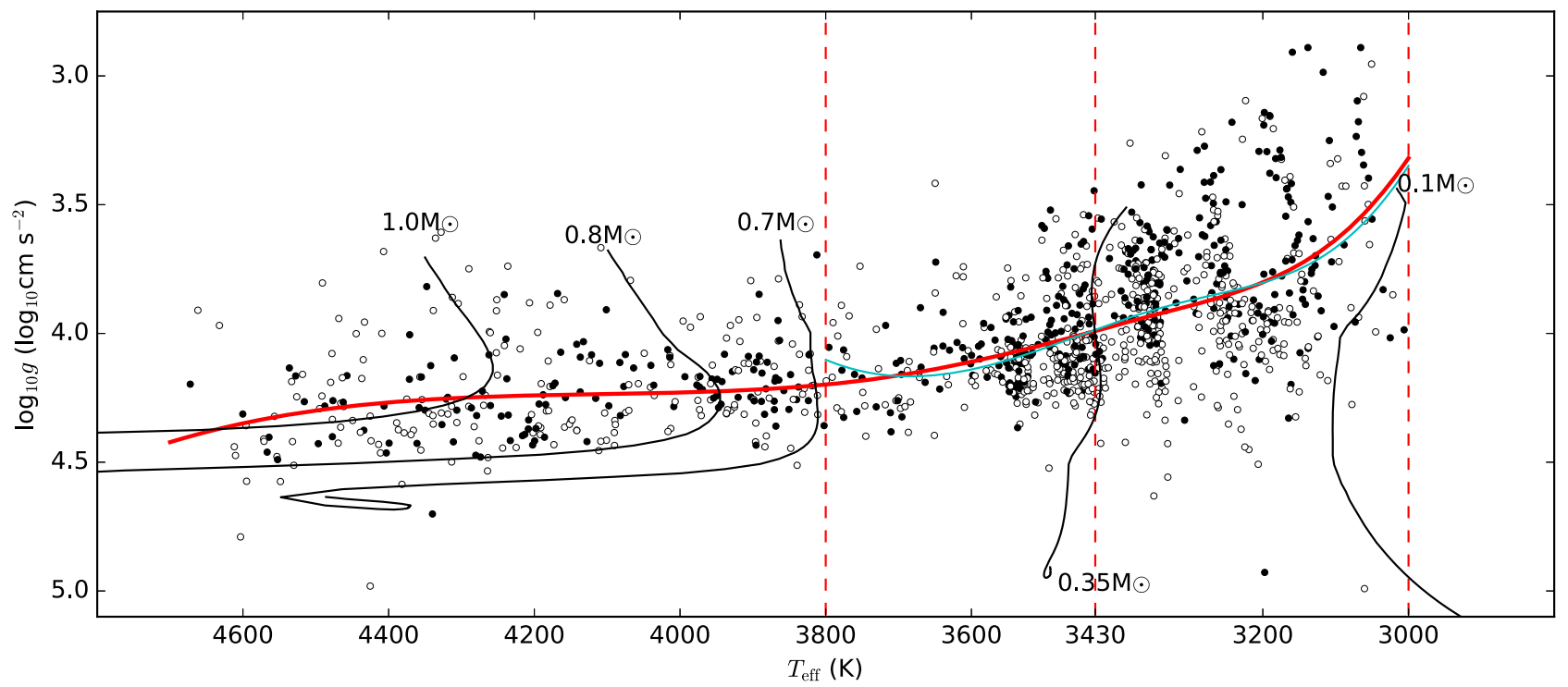

Figure 25. Distribution of spectroscopic $T_{\text {eff }}$ and $\log g$ in Orion A. See Figure 23.

last section, and we adopt the red line as $\log g_{\text {median. }}$. Similar to what we have done in the last section, we also individually look at disk frequency as a function of stellar age in the $0.1-0.7 M_{\odot}$ and $0.1-1.5 M_{\odot}$ mass ranges. Moreover, since our sample in IC 348 is representative down to $0.25 M_{\odot}$, we also make a cut at $0.25 M_{\odot}$. This mass roughly corresponds to $3310 \mathrm{~K}$, which is found by looking at the intersection of the evolutionary track of a $0.25 M_{\odot}$ star and the red line. The result from $0.25-0.7 M_{\odot}$ may be more convincing than that from $0.1-0.25 M_{\odot}$, since our sample is not representative in the latter mass range.

As is shown in the lower panel of Figure 24, in all mass ranges we have seen a slight drop of disk frequency for relatively older stars. The drop is most significant in the $0.25-0.7 M_{\odot}$ range. However, considering the statistical uncertainty, this is also consistent with the same disk frequency for different $\Delta \log g$ bins.

\subsection{Orion $A$}

Similar to the analysis in IC 348, Figure 25 shows the distribution of sources in Orion A on the $\log g-T_{\text {eff }}$ diagram. All of the 1200 sources have $A_{J}<3.0$. Since the Orion A sample is representative down to $\sim 0.35 M_{\odot}$ at this extinction limit, we make a cut at $0.35 M_{\odot}$, which roughly corresponds to $3430 \mathrm{~K}$. The red and cyan lines have the same meaning as in the last section, and we adopt the red as $\log g_{\text {median }}$.

In Figure 26, we show disk frequency as a function of $\Delta \log g$ in the mass ranges of $0.1-1.5 M_{\odot}, 0.7-1.5 M_{\odot}$, $0.35-0.7 M_{\odot}$, and $0.1-0.35 M_{\odot}$. In each mass range, we divide the sample according to $\Delta \log g$ into three bins with an equal number of stars. The definite decrease of disk frequency from $(55.6 \pm 6.7) \%$ to $(41.1 \pm 5.8) \%$, and finally down to $(27.4 \pm 4.7) \%$ in $0.35-0.7 M_{\odot}$ strongly indicates that disk evolution happens, because our sample is representative in this mass range. The absolute disk frequency for $0.1-0.35 M_{\odot}$ suffers from observational bias. However, since $\log g$ and IR excess are two independent measurements, we may not expect that such a bias depends on stellar age. Therefore, the relative disk frequency in the mass range of $0.1-0.35 M_{\odot}$ with different $\Delta \log g$ (from $(55.5 \pm 5.7) \%$ to $(41.9 \pm 4.9) \%$, to

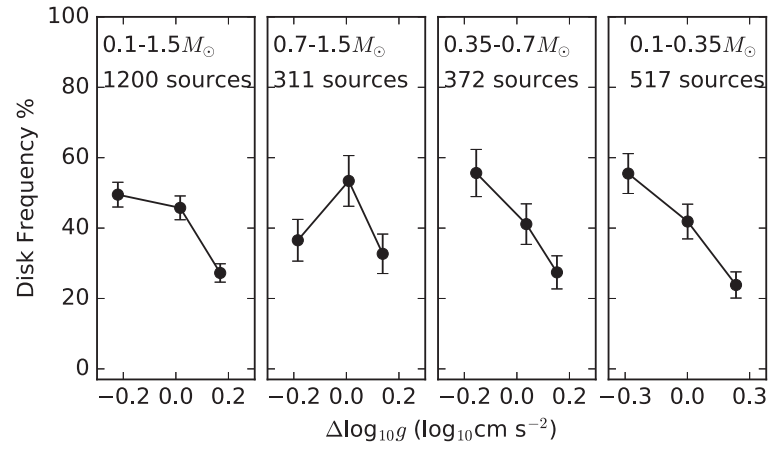

Figure 26. Disk frequency as a function of $\Delta \log g$ (Equation (5)).

$(23.8 \pm 3.7) \%)$ may still provide evidence of disk evolution for the lowest-mass stars.

The trend of disk frequency in the mass bin of $0.7-1.5 M_{\odot}$ is more complicated than other bins-it first goes up, and then goes down as $\Delta \log g$ increases. As mentioned before, in this mass range, an increase in $\Delta \log g$ may indicate older age or lower mass (or both). If the higher $\Delta \log g$ originates from lower mass, then disk frequency should increase for higher $\Delta \log g$, because Section 4.4 and Figure 21 show that disk frequency increases from 1.5 to $0.7 M_{\odot}$. On the other hand, if stellar age is the dominant factor of the variation of surface gravity, then disk frequency should decrease toward higher $\Delta \log g$. We may need to combine both factors to explain our result.

Combining all stars from 0.1 to $1.5 M_{\odot}$ and dividing them into three bins of $\Delta \log g$, we find that disk frequency decreases from $(49.5 \pm 3.5) \%$ to $(45.8 \pm 3.4) \%$, and then to $(27.3 \pm 2.6) \%$. The large number of sources in this cluster as well as its intrinsic spread of age allow us to draw robust conclusion of the detection of intra-cluster disk evolution.

\section{Summary}

Utilizing a sample of stars observed by the IN-SYNC project with accurately derived stellar parameters, we investigate disk evolution in three young clusters. First, in each cluster, averaged over the spread of age, we carefully studied how disk lifetime is dependent on stellar mass for low-mass stars. Previous results 
Table 2

1641 Sources Used in the Sample

\begin{tabular}{|c|c|c|c|c|c|c|c|}
\hline 2MASS designation & $\begin{array}{l}\text { R.A. }^{a} \\
\left(^{\circ}\right)\end{array}$ & $\begin{array}{c}\text { Decl. }^{\mathrm{a}} \\
\left(^{\circ}\right)\end{array}$ & $\begin{array}{l}T_{\text {eff }} \\
(\mathrm{K})\end{array}$ & $\log g$ & $\begin{array}{c}J \\
(\mathrm{mag})\end{array}$ & $\begin{array}{c}H \\
(\mathrm{mag})\end{array}$ & $\begin{array}{c}K_{\mathrm{s}} \\
(\mathrm{mag})\end{array}$ \\
\hline $03281101+3117292$ & 52.04590 & 31.29146 & $3374.6 \pm 8.6$ & $3.99 \pm 0.03$ & $12.441 \pm 0.021$ & $11.457 \pm 0.028$ & $11.028 \pm 0.024$ \\
\hline $03283651+3119289$ & 52.15215 & 31.32471 & $3269.5 \pm 6.2$ & $3.90 \pm 0.04$ & $12.849 \pm 0.020$ & $12.128 \pm 0.026$ & $11.856 \pm 0.023$ \\
\hline $05344929-0518555$ & 83.70540 & -5.31542 & $3459.5 \pm 10.7$ & $4.09 \pm 0.04$ & $12.082 \pm 0.019$ & $11.367 \pm 0.03$ & $11.158 \pm 0.019$ \\
\hline $05332136-0521347$ & 83.33903 & -5.35964 & $4276.0 \pm 200.0$ & $4.28 \pm 0.35$ & $11.253 \pm 0.019$ & $10.332 \pm 0.021$ & $9.769 \pm 0.017$ \\
\hline 0.874 & $10.692 \pm 0.055$ & 0 & 0 & & & & NGC 1333 \\
\hline 0.214 & $11.566 \pm 0.054$ & 0 & 0 & & & & NGC 1333 \\
\hline 1.053 & $8.197 \pm 0.057$ & 1 & 1 & $-1.22 \pm 0.02$ & $1252.01 \pm 21.65$ & $283.65 \pm 6.47$ & NGC 1333 \\
\hline 0.128 & $10.952 \pm 0.052$ & 0 & 1 & $-0.18 \pm 0.05$ & $59.38 \pm 15.92$ & $27.56 \pm 1.83$ & Orion A \\
\hline 0.838 & $8.726 \pm 0.051$ & 1 & 1 & $-1.05 \pm 0.04$ & $867.12 \pm 28.14$ & $265.29 \pm 38.15$ & Orion A \\
\hline
\end{tabular}

Notes.

${ }^{\mathrm{a}}$ R.A. and Decl. given in the 2MASS Point Source Catalog; epoch in J2000.

b Disk type. 0: diskless star; 1: disk-bearing star.

(This table is available in its entirety in machine-readable form.)

show that disk lifetime for intermediate-mass stars is much shorter than that for low-mass stars. This is consistent with our result. However, we think disk lifetime for most stars in the lowmass range are similar in the sense that we do not observe a significant increase of disk frequency from 0.8 to $0.1 \mathrm{M}_{\odot}$.

Second, in each cluster, within a certain mass range, disk frequency (as calculated by the fraction of stars with $4.5 \mu \mathrm{m}$ excess) for stars with larger log $g$ is lower than that with smaller $\log g$, indicating a longer time of disk dispersal for older stars. The result from Orion $\mathrm{A}$ is strong and the most prominent. Evidence of intra-cluster primordial disk evolution in NGC 1333 and IC 348 is still tentative, since the derived frequencies are all compatible within $1 \sigma$. Spectroscopic measurements of $T_{\text {eff }}$ and $\log g$ for more low-mass stars in the two clusters are needed in the future to draw robust evidence of intra-cluster disk evolution. We have demonstrated in this paper the usage of $\log g$ as an age indicator for low-mass stars in the study of disk evolution. This methodology can be applied to other young clusters in the future.

We acknowledge the anonymous referee, Lee Hartmann, Nuria Calvet, and Lynne Hillenbrand for valuable suggestions that greatly improved this manuscript. Stellar parameters for the Perseus sources are derived by Michiel Cottaar. Jonathan B. Foster also contributed to the design of the IN-SYNC program. Special thanks to Jesus Hernández and Mark J. Pecaut for patiently answering questions about details and techniques in their work. Y.Y. acknowledges China Scholarship Council (CSC) for supporting this research.

\section{Appendix}

In Table 2, we provide information of the 1641 sources used in our sample, including 70 in NGC 1333, 239 in IC 34, and 1332 in the Orion A molecular cloud. Apart from stellar parameters and photometric data, we also indicate whether hydrogen emission lines in the Brackett series $\left(n^{\prime}=4\right)$ can be observed in their $H$-band APOGEE spectra. Whenever brackett emission lines can be identified, we also present the equivalent width (EW), line flux, and full-width half-maximum (FWHM) of the Br11 line at $1681 \mathrm{~nm}$ - the strongest Brackett line in the APOGEE wavelength range.

It has been long known that hydrogen emission lines are indicative of large-scale gas flows. Generally speaking, the profiles of Br11 show complex behaviors: most exhibit lines that are symmetric about line center; while some show significant blueward asymmetry or redshifted absorption (e.g., $2 \mathrm{M} 03292187+3115363$ in NGC 1333), which are direct evidence of mass infall (Edwards et al. 1994); a few stars (e.g., 2M05341221-0450072 in Orion A) have overlying absorption on top of the emission profile. Just like other highexcitation hydrogen emission lines (Folha et al. 1997; Muzerolle et al. 1998), blueshifted absorption commonly seen in $\mathrm{H} \alpha$ can seldom be observed.

In the densest region of $\mathrm{ONC}$, stellar spectra are contaminated by narrow $\left(\mathrm{FWHM}<70 \mathrm{~km} \mathrm{~s}^{-1}\right)$ nebula emission due to imperfect sky subtraction; however, in our sample, the Br11 emission line with large line widths (FWHM $>100 \mathrm{~km} \mathrm{~s}^{-1}$ ) are only found in stars exhibiting infrared excess. Models with infalling gas via magnetospheric accretion have successfully reproduced these characteristics (Muzerolle et al. 1998, 2001). Others may in the future like to model these line profiles.

\section{ORCID iDs}

Yuhan Yao (iD https://orcid.org/0000-0001-6747-8509 Michael R. Meyer (iD https://orcid.org/0000-0003-1227-3084 Kevin R. Covey (iD https://orcid.org/0000-0001-6914-7797

\section{References}

Allard, F., Homeier, D., \& Freytag, B. 2012, RSPTA, 370, 2765

Allen, L. E., Calvet, N., D’Alessio, P., et al. 2004, ApJS, 154, 363

Andrews, S. M., Rosenfeld, K. A., Kraus, A. L., \& Wilner, D. J. 2013, ApJ, 771,129

Baraffe, I., Chabrier, G., Allard, F., \& Hauschildt, P. H. 1998, A\&A, 337, 403 Bowler, B. P. 2016, PASP, 128, 102001

Cardelli, J. A., Clayton, G. C., \& Mathis, J. S. 1989, ApJ, 345, 245

Carpenter, J. M., Mamajek, E. E., Hillenbrand, L. A., \& Meyer, M. R. 2006, ApJL, 651, L49

Cohen, M., Wheaton, W. A., \& Megeath, S. T. 2003, AJ, 126, 1090 
Cottaar, M., Covey, K. R., Foster, J. B., et al. 2015, ApJ, 807, 27

Cottaar, M., Covey, K. R., Meyer, M. R., et al. 2014, ApJ, 794, 125

Currie, T., Lada, C. J., Plavchan, P., et al. 2009, ApJ, 698, 1

Da Rio, N., Robberto, M., Hillenbrand, L. A., Henning, T., \& Stassun, K. G. 2012, ApJ, 748, 14

Da Rio, N., Tan, J. C., Covey, K. R., et al. 2016, ApJ, 818, 59

Dahm, S. E., \& Hillenbrand, L. A. 2007, AJ, 133, 2072

D’Alessio, P., Calvet, N., Hartmann, L., Franco-Hernández, R., \& Servín, H. 2006, ApJ, 638, 314

Edwards, S., Hartigan, P., Ghandour, L., \& Andrulis, C. 1994, AJ, 108, 1056 Eisenstein, D. J., Weinberg, D. H., Agol, E., et al. 2011, AJ, 142, 72

Evans, N. J., II, Allen, L. E., Blake, G. A., et al. 2003, PASP, 115, 965

Evans, N. J., II, Dunham, M. M., Jørgensen, J. K., et al. 2009, ApJS, 181, 321

Fang, M., Kim, J. S., van Boekel, R., et al. 2013, ApJS, 207, 5

Fedele, D., van den Ancker, M. E., Henning, T., Jayawardhana, R., \& Oliveira, J. M. 2010, A\&A, 510, A72

Flaherty, K. M., Pipher, J. L., Megeath, S. T., et al. 2007, ApJ, 663, 1069

Folha, D., Emerson, J., \& Calvet, N. 1997, in IAU Symp. 182, Low Mass Star Formation from Infall to Outflow (Poster Proceedings), ed. F. Malbet \& A. Castets (Grenoble: Obs. Grenoble), 272

Foster, J. B., Cottaar, M., Covey, K. R., et al. 2015, ApJ, 799, 136

Gaia Collaboration, Brown, A. G. A., Vallenari, A., et al. 2018, A\&A, 616, A1

Greene, T. P., Wilking, B. A., Andre, P., Young, E. T., \& Lada, C. J. 1994, ApJ, 434, 614

Gutermuth, R. A., Myers, P. C., Megeath, S. T., et al. 2008, ApJ, 674, 336

Haisch, K. E., Jr., Lada, E. A., \& Lada, C. J. 2001, ApJL, 553, L153

Hartigan, P., Edwards, S., \& Ghandour, L. 1995, ApJ, 452, 736

Hartmann, L. 2009, Accretion Processes in Star Formation (2nd ed; Cambridge: Cambridge Univ. Press)

Hayashi, C. 1961, PASJ, 13, 450

Hernández, J., Calvet, N., Briceño, C., et al. 2007, ApJ, 671, 1784

Hernández, J., Calvet, N., Hartmann, L., et al. 2005, AJ, 129, 856

Hernández, J., Hartmann, L., Megeath, T., et al. 2007, ApJ, 662, 1067

Hillenbrand, L. A., Strom, S. E., Calvet, N., et al. 1998, AJ, 116, 1816

Hsu, W.-H., Hartmann, L., Allen, L., et al. 2012, ApJ, 752, 59

Hsu, W.-H., Hartmann, L., Allen, L., et al. 2013, ApJ, 764, 114

Johnson, J. A., Aller, K. M., Howard, A. W., \& Crepp, J. R. 2010, PASP, 122,905

Kennedy, G. M., \& Kenyon, S. J. 2009, ApJ, 695, 1210

Kryukova, E., Megeath, S. T., Gutermuth, R. A., et al. 2012, AJ, 144, 31

Lada, C. J., Alves, J., \& Lada, E. A. 1996, AJ, 111, 1964

Lada, C. J., Muench, A. A., Luhman, K. L., et al. 2006, AJ, 131, 1574

Lloyd, S. P. 1982, ITIT, 28, 129
Luhman, K. L., Esplin, T. L., \& Loutrel, N. P. 2016, ApJ, 827, 52

Luhman, K. L., Hernández, J., Downes, J. J., Hartmann, L., \& Briceño, C. 2008, ApJ, 688, 362

Luhman, K. L., Stauffer, J. R., Muench, A. A., et al. 2003, ApJ, 593, 1093

Majewski, S. R., Schiavon, R. P., Frinchaboy, P. M., et al. 2017, AJ, 154, 94

Mamajek, E. E. 2009, in AIP Conf. Ser. 1158, Exoplanets and Disks: Their Formation and Diversity, ed. T. Usuda, M. Tamura, \& M. Ishii (Meliville, NY: AIP), 3

Megeath, S. T., Gutermuth, R., Muzerolle, J., et al. 2012, AJ, 144, 192

Meyer, M. R. 2009, in IAU Symp. 4, Circumstellar Disk Evolution: Constraining Theories of Planet Formation, ed. E. E. Mamajek, D. R. Soderblom, \& R. F. G. Wyse (Cambridge: Cambridge Univ. Press), 111

Meyer, M. R., Backman, D. E., Weinberger, A. J., \& Wyatt, M. C. 2007, in Protostars and Planets V, ed. B. Reipurth, D. Jewitt, \& K. Keil (Tucson, AZ: Univ. Arizona Press), 573

Meyer, M. R., Calvet, N., \& Hillenbrand, L. A. 1997, AJ, 114, 288

Muench, A., Getman, K., Hillenbrand, L., \& Preibisch, T. 2008, in Handbook of Star Forming Regions, Volume I: The Northern Sky, ed. B. Reipurth (San Francisco, CA: ASP), 483

Muench, A. A., Lada, C. J., Luhman, K. L., Muzerolle, J., \& Young, E. 2007, AJ, 134, 411

Muzerolle, J., Calvet, N., \& Hartmann, L. 1998, ApJ, 492, 743

Muzerolle, J., Calvet, N., \& Hartmann, L. 2001, ApJ, 550, 944

Muzerolle, J., Hartmann, L., \& Calvet, N. 1998, AJ, 116, 455

Pecaut, M. J., \& Mamajek, E. E. 2013, ApJS, 208, 9

Press, W. H., Teukolsky, S. A., Vetterling, W. T., \& Flannery, B. P. 2002, in Numerical Recipes in $\mathrm{C}++$ : The Art of Scientific Computing, ed. H. William (2nd ed.; Cambridge: Cambridge Univ. Press), 1002

Ribas, Á, Bouy, H., \& Merín, B. 2015, A\&A, 576, A52

Ribas, Á, Merín, B., Bouy, H., \& Maud, L. T. 2014, A\&A, 561, A54

Robitaille, T. P., Whitney, B. A., Indebetouw, R., Wood, K., \& Denzmore, P. 2006, ApJS, 167, 256

Siess, L., Dufour, E., \& Forestini, M. 2000, A\&A, 358, 593

Skrutskie, M. F., Cutri, R. M., Stiening, R., et al. 2006, AJ, 131, 1163

Wainscoat, R. J., Cohen, M., Volk, K., Walker, H. J., \& Schwartz, D. E. 1992, ApJS, 83, 111

Wilking, B. A., Meyer, M. R., Greene, T. P., Mikhail, A., \& Carlson, G. 2004, AJ, 127, 1131

Williams, J. P., \& Cieza, L. A. 2011, ARA\&A, 49, 67

Yasui, C., Kobayashi, N., Tokunaga, A. T., \& Saito, M. 2014, MNRAS, 442, 2543

Young, K. E., Young, C. H., Lai, S.-P., Dunham, M. M., \& Evans, N. J., II 2015, AJ, 150, 40 